\title{
Gapless Symmetry-Protected Topological Order
}

\author{
Thomas Scaffidi, ${ }^{1, *}$ Daniel E. Parker, ${ }^{1, \dagger}$ and Romain Vasseur ${ }^{1,2,3, *}$ \\ ${ }^{1}$ Department of Physics, University of California, Berkeley, California 94720, USA \\ ${ }^{2}$ Materials Sciences Division, Lawrence Berkeley National Laboratory, Berkeley, California 94720, USA \\ ${ }^{3}$ Department of Physics, University of Massachusetts, Amherst, Massachusetts 01003, USA \\ (Received 15 May 2017; revised manuscript received 19 September 2017; published 29 November 2017)
}

\begin{abstract}
We introduce exactly solvable gapless quantum systems in $d$ dimensions that support symmetryprotected topological (SPT) edge modes. Our construction leads to long-range entangled, critical points or phases that can be interpreted as critical condensates of domain walls "decorated" with dimension $(d-1)$ SPT systems. Using a combination of field theory and exact lattice results, we argue that such gapless SPT systems have symmetry-protected topological edge modes that can be either gapless or symmetry broken, leading to unusual surface critical properties. Despite the absence of a bulk gap, these edge modes are robust against arbitrary symmetry-preserving local perturbations near the edges. In two dimensions, we construct wave functions that can also be interpreted as unusual quantum critical points with diffusive scaling in the bulk but ballistic edge dynamics.
\end{abstract}

DOI: 10.1103/PhysRevX.7.041048

\section{INTRODUCTION}

An overarching goal of condensed-matter physics is to identify and classify new phases of matter. Since probing a system amounts to perturbing it and measuring how it reacts, understanding the physics of a phase reduces to the problem of identifying the low-lying excitations that perturbations can create. A natural dichotomy is to distinguish gapless phases, which possess excitations arbitrarily close to the ground state, from gapped ones, which have a finite spectral gap in the thermodynamic limit. Naively, this would suggest gapped systems are featureless at low energy.

Discoveries in recent decades have shown that the story is more subtle, as a large class of gapped phases can host gapless excitations localized to edges and defects. Such excitations are protected by a combination of symmetries and the topological properties of the bulk system. These topological phases include long-range entangled systems $[1,2]$ with intrinsic topological order and bulk anyonic excitations, such as quantum Hall states or spin liquids [3]. They can be further enriched by symmetries [4-10]. Following the theoretical prediction and subsequent experimental discovery of topological insulators and superconductors [11-20], attention has turned to short-range

\footnotetext{
*thomas.scaffidi@berkeley.edu

†daniel_parker@berkeley.edu

rvasseur@umass.edu
}

Published by the American Physical Society under the terms of the Creative Commons Attribution 4.0 International license. Further distribution of this work must maintain attribution to the author(s) and the published article's title, journal citation, and DOI.
Subject Areas: Condensed Matter Physics, Magnetism, Topological Insulators entangled phases with topological edge modes protected by symmetry [21-30]. These symmetry-protected topological (SPT) phases may be realized in strongly interacting systems, like the experimentally accessible Haldane phase in quantum spin chains [31]. This shift in paradigm from a band topology analysis of noninteracting Hamiltonians $[32,33]$ to strongly correlated systems led to the development of nonperturbative techniques, resulting in an essentially exhaustive classification of gapped bosonic $[27,29,30,34]$ and, to some extent, fermionic SPT phases [24,35-39]. All these phases enjoy a bulk spectral gap, and indeed, this gap often plays a crucial role in understanding topological phases.

Must systems have a bulk gap to possess the properties of topological phases? Given the prevalence of gapless systems in nature, it is possible that many of the features ascribed to gapped topological systems are "hidden" around their edges [40]. As an example of a step in this direction, it was recently argued that topological phases can survive in nonequilibrium, highly excited states where there is no notion of a gap [41-43]. In the less-exotic realm of equilibrium physics at low temperatures, Weyl and Dirac semimetals with topologically protected Fermi arc surface states [44] are gapless systems with topological properties that have been experimentally confirmed in several materials [45-47]. Other examples related to free-fermionic systems include the A phase of superfluid ${ }^{3} \mathrm{He}$ [48], power-law superconducting chains [49-52], and recent proposals for gapless topological insulators [53] and superconductors [54,55].

Examples of gapless topological systems [40] are, for the most part, restricted to noninteracting systems. Some exceptions include topological Mott insulators [56], topological Luttinger liquids [57,58], gapless spin liquids $[4,59]$, 
the Gaffnian quantum Hall state [60], and the composite Fermi liquids in the half-filled Landau level [61,62]. However, the precise topological nature-and edge properties - of many of these systems remains controversial.

In this work, we present a general construction of strongly interacting, long-range entangled, quantum systems that are gapless in the bulk with topological edge modes protected by symmetry. These gapless symmetryprotected topological states of matter are generated via a systematic procedure that employs standard tools of gapped SPT phases, making their topological properties transparent. For conciseness, we refer to them as "gapless SPTs" (gSPTs). Just as normal SPTs can be thought of as "twisted" paramagnets, gapless SPTs can be obtained by twisting ordinary quantum critical points or critical phases. Some examples of gapless SPTs may be produced starting from an SPT and tuning a subset of the degrees of freedom (d.o.f.) to criticality.

In Sec. II, we outline the general construction based on the decorated domain-wall picture of gapped SPT phases [63]. This picture yields many examples, but we focus on several of them with the virtue of being exactly solvable: a topological critical Ising chain and a topological Luttingerliquid phase in one dimension (Sec. III), and a topological gapless spin liquid in two dimensions (Sec. IV). In all cases, we start with the parent Hamiltonian, find the exact ground-state wave function, and demonstrate the presence of topologically protected edge modes that must be either gapless or symmetry broken. Despite the absence of a bulk gap, the topological edge modes in such gSPT systems are robust to arbitrary symmetry-preserving boundary perturbations and require no fine-tuning beyond closing the bulk gap. In particular, our general construction can be applied to both quantum critical points and gapless phases.

The topological edge modes of gSPTs can be interpreted as giving rise to exotic surface criticality [64]. Below, we show that this can take the form of anomalous edge magnetization or the appearance of ballistic dynamics at the edge of a diffusive system. Our construction therefore yields a host of gapless systems that blend the physics of quantum critical and topological systems.

\section{GENERAL CONSTRUCTION}

Consider a bosonic system in $d$ dimensions composed of $\sigma$ and $\tau$ d.o.f. and symmetry group $G_{\sigma} \times G_{\tau}$ with $G_{\sigma}=\mathbb{Z}_{2}$. Our construction starts from the decorated domain-wall picture of SPTs [63]. In this picture, a "trivial" disordered phase ["trivial" paramagnets, Fig. 1(a)] is thought of as a gapped condensate of domain walls. Nontrivial SPT phases ["topological" paramagnets, Fig. 1(b)] are produced by "decorating" the domain walls of $G_{\sigma}=\mathbb{Z}_{2}$ with $(d-1)$ dimensional SPT phases protected by the symmetry $G_{\tau}$. The protected edge modes appear naturally: Domain walls that end at a boundary carry the topologically protected edge mode of the lower-dimensional SPT. (a) Trivial

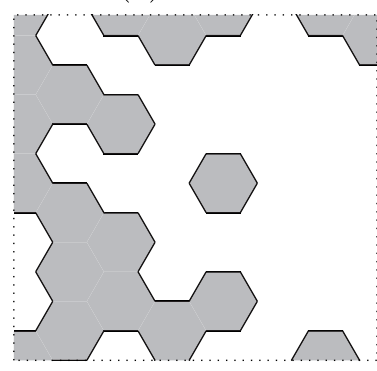

(b) SPT
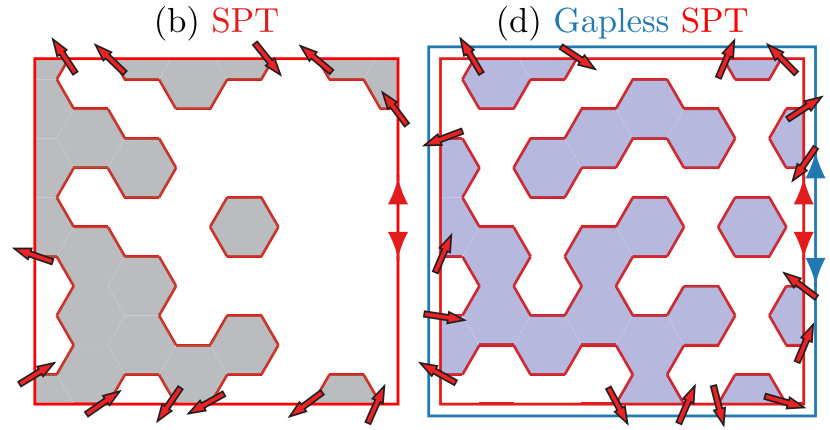

(c) Gapless Trivial

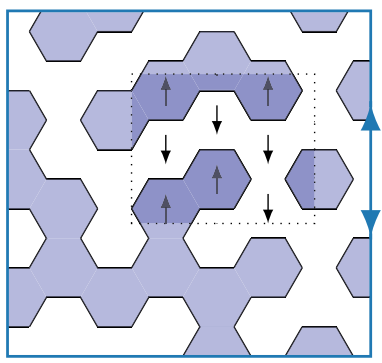

(d) Gapless SPT
FIG. 1. Representative states of each order in the $2 d$ example. (a) Trivial: Paramagnetic spins on a triangular lattice with fluctuating domain walls. (b) SPT: Decorating the domain walls, giving a SPT with a $c=1$ edge mode. (c) Gapless trivial: Tuning the domain walls to criticality by restricting them to fully packed loop configurations (defined below), closing the gap and giving a $c=1$ edge mode. (d) Doing both yields a gapless SPT with $c=1+1=2$ edge modes.

To make a gapless system, we tune the domain-wall condensate to criticality (i.e., tune the underlying $\sigma$ d.o.f. to criticality). When the domain walls are not decorated [the "gTrivial" case, Fig. 1(c)], this typically tunes the system to an ordinary quantum critical point. For example, in $1 d$, one can consider the domain walls of a critical Ising chain, and in $2 d$, one can use the domain walls of an Ising frustrated antiferromagnet. Generically, there is nothing protected about the edge of such gTrivial systems: They may or may not have additional gapless modes at their boundaries.

The crucial step is that one may decorate the gTrivial system with lower-dimensional SPT systems. This leads to a topologically distinct gapless state [called "gSPT," Fig. 1(d)], which, in analogy to the gapped case, has the same properties as gTrivial in the bulk but completely different edge physics. Topologically protected edge modes appear in gSPT that can be gapped out only at the price of breaking the symmetry at the edge (either spontaneously or explicitly). In short, starting from a gapped SPT, one can generate a gapless SPT by making the domain-wall condensate critical while keeping the same domain-wall decoration.

The resulting gSPT systems are tuned to criticality in the bulk, while the edge modes are robust against symmetrypreserving perturbations acting near the edge. Even though 
some of the examples we treat in this work correspond to critical points, as opposed to gapless phases, this is by no means a limitation of our construction. (To be clear, gapless SPTs are not "symmetry-protected gapless phases" [65-67] - the gaplessness of the bulk theory is not protected by symmetry.) As we show explicitly in Sec. III C, the same construction of applying the SPT decoration can be performed in gapless phases, such as Luttinger liquids in $1 d$ [57] or gapless spin liquids in 3D [68], to obtain gapless SPT phases. More generally, gSPTs are as stable as their underlying gTrivial states before applying the decoration. In particular, gSPTs have exactly the same spectrum as their parent gTrivial systems on closed manifolds since they are related by a local unitary transformation.

\section{ONE DIMENSION}

This section provides a first example of a gapless SPT in one dimension, combining the features of a wellunderstood $1 d$ gapped SPT and of the critical Ising model. Starting from a gapped SPT with $\mathbb{Z}_{2} \times \mathbb{Z}_{2}$ symmetry, we bring one of the spin species to criticality and argue, in this exactly solvable limit, that the gapless system has topological edge modes. Going beyond this exactly solvable limit, we numerically demonstrate the robustness of the symmetry-protected topological edge modes against arbitrary symmetry-preserving perturbations.

\section{A. Gapped $\mathbb{Z}_{2} \times \mathbb{Z}_{2}$ SPT}

To set the notation, we first recall the construction of a gapped SPT with $\mathbb{Z}_{2} \times \mathbb{Z}_{2}$ symmetry in one dimension $[30,43,63,69]$, which is closely related to the experimentally observable Haldane phase $[31,70,71]$. Consider a spin$1 / 2$ chain with two alternating spin species: $\sigma$ (on sites $i$ ) and $\tau$ (on sites $i+\frac{1}{2}$ ), as shown in Fig. 2. We impose an inviolable $\mathbb{Z}_{2} \times \mathbb{Z}_{2}=G_{\sigma} \times G_{\tau}$ global symmetry generated by $\mathcal{C}_{\sigma}=\prod_{i} \sigma_{i}^{x}$ and $\mathcal{C}_{\tau}=\prod_{i} \tau_{i+\frac{1}{2}}^{x}$. In $1 d, \mathbb{Z}_{2} \times \mathbb{Z}_{2}$ is the minimal symmetry required to have a nontrivial SPT.

A trivial paramagnetic phase is obtained with the zerocorrelation length Hamiltonian

$$
H_{\text {Trivial }}=-\sum_{i} \sigma_{i}^{x}+\tau_{i-\frac{1}{2}}^{x}
$$

with ground-state wave function

$$
\left|\Psi_{\text {Trivial }}\right\rangle=\sum_{\left\{\sigma^{z}\right\},\left\{\tau^{z}\right\}}\left|\sigma^{z}, \tau^{z}\right\rangle,
$$

where the sum runs over all $\sigma^{z}$ and $\tau^{z}$ configurations. This can be thought of as a gapped phase where domain walls have "proliferated."

An exactly solvable example of a nontrivial SPT phase can then be made by "twisting" or "decorating" this Hamiltonian by a local unitary operator $U_{1 d}$ [30]. Define
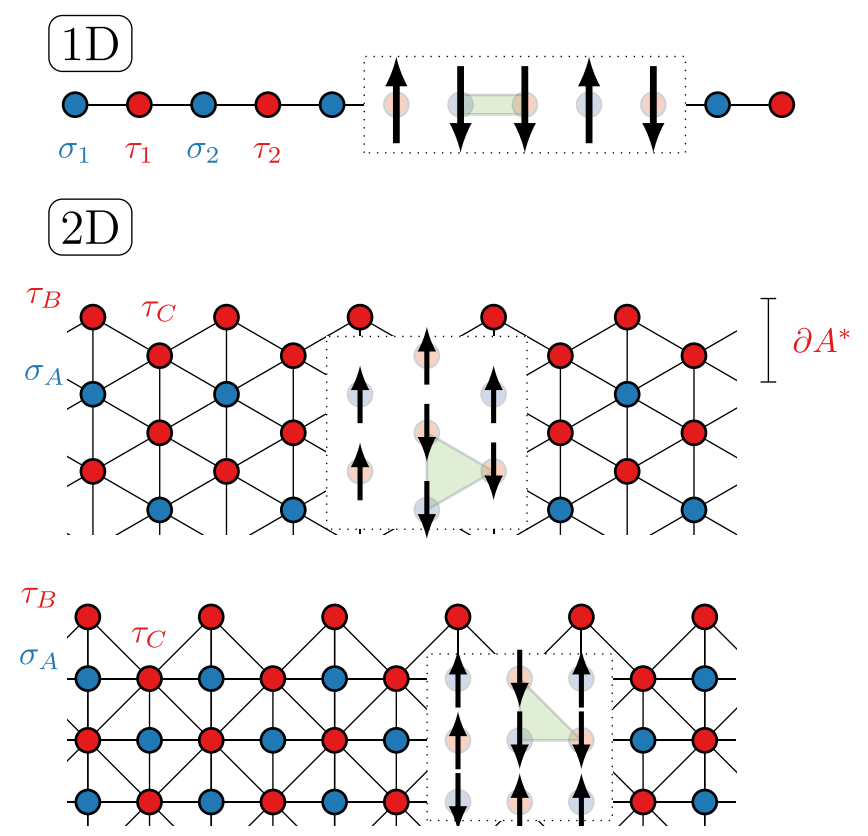

FIG. 2. Lattices used throughout this paper to construct SPT and gSPT wave functions in one (top) and two dimensions (bottom: triangular and Union Jack lattices). The control- $Z$ twist operator used to obtain nontrivial SPT order gives a factor of $(-1)$ to links with two down spins in the $1 d$ case and triangles with three down spins in the $2 d$ case, as exemplified by the green shading.

$$
U_{1 d}=\prod_{i} \mathrm{CZ}_{i-1, i-1 / 2} \mathrm{CZ}_{i, i-1 / 2}
$$

where $\mathrm{CZ}_{i j}=(-1)^{\delta_{\downarrow}}$ is the control- $Z$ two-qubit operator with $\delta_{\downarrow \downarrow}=\frac{1}{4}\left(1-\sigma_{i}^{z}\right)\left(1-\tau_{j}^{z}\right)$, which gives a factor of $(-1)$ if the two spins are down and a +1 otherwise-see Fig. 2. Alternatively, $U_{1 d}$ can be thought of as attaching charges of one $\mathbb{Z}_{2}$ symmetry to domain walls of the other $\mathbb{Z}_{2}$ symmetry [72]. Under periodic boundary conditions, this unitary transformation commutes with the $\mathbb{Z}_{2} \times \mathbb{Z}_{2}$ symmetry. Explicitly, the nontrivial SPT Hamiltonian $H_{\text {SPT }}=U_{1 d} H_{\text {Trivial }} U_{1 d}$ reads

$$
H_{\mathrm{SPT}}=-\sum_{i} \tau_{i-\frac{1}{2}}^{z} \sigma_{i}^{x} \tau_{i+\frac{1}{2}}^{z}+\sigma_{i-1}^{z} \tau_{i-\frac{1}{2}}^{x} \sigma_{i}^{z}
$$

with ground-state wave function

$$
\left|\Psi_{\mathrm{SPT}}\right\rangle=U_{1 d}\left|\Psi_{\text {Trivial }}\right\rangle=\sum_{\left\{\sigma^{z}\right\},\left\{\tau^{z}\right\}} e^{i \theta_{1 d}\left(\sigma^{z}, \tau^{z}\right)}\left|\sigma^{z}, \tau^{z}\right\rangle,
$$

with $e^{i \theta_{1 d}\left(\sigma^{z}, \tau^{z}\right)}=\prod(-1)^{\delta_{\downarrow \downarrow}}$. The fact that $H_{\text {Trivial }}$ and $H_{\mathrm{SPT}}$ lie in different SPT phases means that transforming one continuously into the other must either break the $\mathbb{Z}_{2} \times \mathbb{Z}_{2}$ symmetry or close the gap. Both Hamiltonians are short-range entangled, gapped paramagnets, and they have the same spectrum with periodic boundary conditions. 
However, with open boundary conditions, they differ at the edge: $H_{\mathrm{SPT}}$ has spin-1/2 gapless edge excitations. We emphasize that the edge modes are topologically protected: They remain when arbitrary perturbations are added to Eq. (4), as long as the $\mathbb{Z}_{2} \times \mathbb{Z}_{2}$ symmetry is preserved.

\section{B. Gapless $\mathbb{Z}_{2} \times \mathbb{Z}_{2}$ SPT}

Starting from the trivial paramagnet of Eq. (1), one can drive the system to criticality by adding a ferromagnetic interaction for the $\sigma$ spins. This can also be interpreted as driving the domain walls of $G_{\sigma}$ to criticality. Explicitly,

$$
H_{\text {gTrivial }}=H_{\text {Trivial }}-\sum_{i} \sigma_{i}^{z} \sigma_{i-1}^{z} .
$$

This is a critical Ising chain for $\sigma$ and a trivial paramagnet for $\tau$. At low energy, one can ignore the gapped $\tau$ d.o.f., and the criticality is in the Ising universality class.

Using the same local unitary $U_{1 d}$ as above, we define a gapless SPT system as

$$
H_{\mathrm{gSPT}}=U_{1 d} H_{\mathrm{gTrivial}} U_{1 d}=H_{\mathrm{SPT}}-\sum_{i} \sigma_{i}^{z} \sigma_{i-1}^{z} .
$$

We show that, just as with $H_{\text {Trivial }}$ and $H_{\mathrm{SPT}}, H_{\mathrm{gTrivial}}$ and $H_{\mathrm{gSPT}}$ have the same bulk properties but differ at the edge. Namely, $H_{\mathrm{gSPT}}$ supports topological edge modes. This difference can also be interpreted as a difference of (conformally invariant) boundary conditions for the Ising conformal field theory (CFT): The edge modes of $H_{\mathrm{gSPT}}$ effectively lead to fixed boundary conditions (whereby the spins at the edge are held fixed, either up or down), while $H_{\mathrm{g} T r i v i a l}$ has a free boundary condition. Note that fixed boundary conditions for an Ising CFT normally require the symmetry to be explicitly broken at the edge. Obtaining such boundary conditions for an Ising-symmetric Hamiltonian is therefore highly unusual and a signature of the anomalous character of the boundary properties of $H_{\mathrm{gSPT}}$.

To see how this comes about, consider the exactly solvable case of $H_{\mathrm{gSPT}}$ on a semi-infinite chain $i \geq 0$ starting with $\sigma_{0}$. (For both $H_{\mathrm{SPT}}$ and $H_{\mathrm{gSPT}}$, the term $\sigma_{0}^{x} \tau_{1 / 2}$ is disallowed by symmetry, so we start with $\sigma_{0}^{z} \sigma_{1}^{z}+\sigma_{0}^{z} \tau_{1 / 2}^{x} \sigma_{1}^{z}$.) This Hamiltonian has two exactly degenerate ground states indexed by the edge mode $\sigma_{0}^{z}= \pm 1$, denoted $\left|\Psi_{\mathrm{gSPT}}\right\rangle_{ \pm}$. One easily finds that

$$
\left|\Psi_{\mathrm{gSPT}}\right\rangle_{ \pm}=U_{1 d}\left(|\operatorname{Ising}\rangle_{ \pm} \otimes \sum_{\left\{\tau^{z}\right\}}\left|\tau^{z}\right\rangle\right)
$$

where $U_{1 d}$ is the unitary defined above restricted to $i>0$ and where $\mid$ Ising $\rangle_{ \pm}$are the critical Ising ground states for the $\sigma$ d.o.f. with fixed boundary spin $\sigma_{0}^{z}= \pm \frac{1}{2}$. Since $U_{1 d}$ commutes with $\sigma_{i}^{z}$, the magnetization $m_{i} \equiv\left\langle\sigma_{i}^{z}\right\rangle$ can be computed for the state $\mid$ Ising $\rangle_{ \pm}$, for which it is known to decay as $x^{-1 / 8}$, where $x \propto i$ is the distance from the edge [73]. Of course, the wave functions (8) break the $G_{\sigma}=\mathbb{Z}_{2}$ symmetry at the boundary, and the true ground states will be symmetry-preserving cat states $\left|\Psi_{\mathrm{gSPT}}\right\rangle_{+} \pm\left|\Psi_{\mathrm{gSPT}}\right\rangle_{-}$. However, as in regular symmetry breaking, a minute boundary field $h_{B} \sigma_{0}^{z}$ (or bulk field $h \sum_{i} \sigma_{i}^{z}$ ) is enough to pick either $\left|\Psi_{\mathrm{gSPT}}\right\rangle_{+}$or $\left|\Psi_{\mathrm{gSPT}}\right\rangle_{-}$, thereby leading to a nonzero magnetization that decays into the bulk as $x^{-1 / 8}$.

This result is in stark contrast to the gTrivial case where the boundary condition is free, the ground state is nondegenerate, and the magnetization is zero, both at the edge and in the bulk [74]. Note that the bulk magnetization $m_{\text {bulk }}=(1 / L) \sum_{i} m_{i}$ also vanishes for gSPT in the limit $L \rightarrow \infty$, although very slowly: $m_{\text {bulk }} \sim L^{-1 / 8}$ ( $L$ is the system size).

Using standard density matrix renormalization group (DMRG) techniques [75,76], we numerically compare the typical magnetization profile for gSPT and gTrivial systems with open boundary conditions. We include small but arbitrary symmetry-preserving boundary perturbations and a small $g_{\tau} \sum_{i} \tau_{i-1 / 2}^{z} \tau_{i+1 / 2}^{z}$ term that gives a nonzero correlation length to the gapped $\tau$ spins. In the presence of a magnetic field much smaller than the CFT finite-size gap, we find a clear qualitative difference between gSPT and gTrivial systems (Fig. 3).

The properties of $H_{\mathrm{gSPT}}$ are robust and not a product of fine-tuning. They are stable in the presence of any symmetry-preserving perturbations, as long as the $\tau$ gap is not closed and the $\sigma$ spins remain critical. The entire phase boundary between the nontrivial SPT (paramagnet) to a ferromagnet has the character of a gSPT, and we expect our conclusions to broadly apply to more general phase transitions between SPT and broken-symmetry phases.

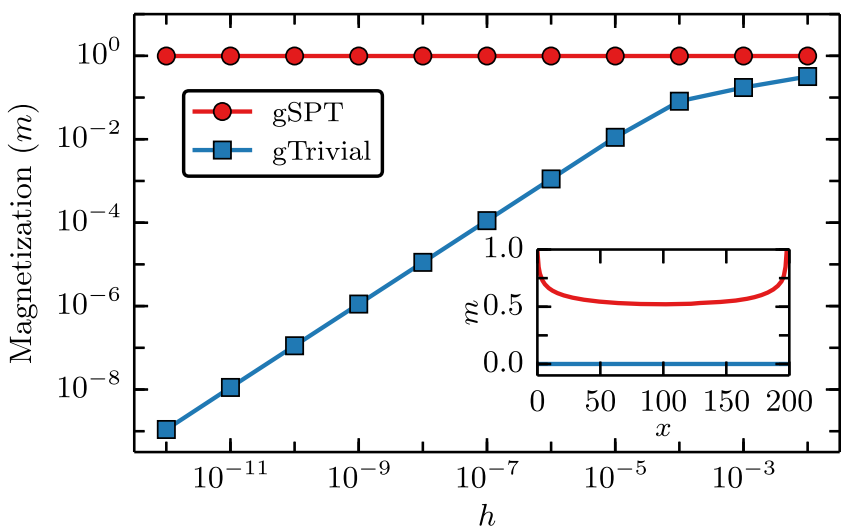

FIG. 3. Edge magnetization of the critical $\sigma$ spins for typical gSPT and gTrivial ground states as a function of a small magnetic field. The ground states were computed on $L=200 \sigma$ spins (and $200 \tau$ spins) using DMRG, including small but arbitrary symmetry-preserving boundary perturbations. Inset: Spatial magnetization profiles for a field $h=10^{-10}$. 
We add several types of perturbations to $H_{\mathrm{gSPT}}$ and consider the generalized Hamiltonian

$$
H_{\mathrm{gSPT}}^{\prime}=U_{1 d} H_{\mathrm{gTrivial}}^{\prime} U_{1 d}+\delta\left(\sigma_{0}^{x}+\tau_{\frac{1}{2}}^{x}+\sigma_{L-1}^{x}+\tau_{L-\frac{1}{2}}^{x}\right)
$$

where

$$
\begin{aligned}
H_{\mathrm{gTrivial}}^{\prime}= & -\sum_{i} \sigma_{i}^{x}+g_{\sigma} \sigma_{i}^{z} \sigma_{i+1}^{z}+u_{\sigma} \sigma_{i}^{x} \sigma_{i+1}^{x} \\
& -\sum_{i} \Delta_{\tau}\left(\tau_{i-\frac{1}{2}}^{x}+g_{\tau} \tau_{i-\frac{1}{2}}^{z} \tau_{i+\frac{1}{2}}^{z}+u_{\tau} \tau_{i-\frac{1}{2}}^{x} \tau_{i+\frac{1}{2}}^{x}\right) \\
& +\sum_{i} \gamma \sigma_{i}^{x} \tau_{i+\frac{1}{2}}^{x} .
\end{aligned}
$$

Here, $\delta$ parametrizes additional terms at the edges, $g_{\tau}$ gives a nonzero correlation length for the $\tau$ spins, $u_{\sigma}$ and $u_{\tau}$ are interaction terms for the $\sigma$ and $\tau$ spins to take them away from integrable points, and $\gamma$ couples the $\sigma$ and $\tau$ sectors. The parameter $\Delta_{\tau}$ controls the gap of the $\tau$ spins, which is used to improve finite-size convergence in exact diagonalization (ED). We choose the parameters $u_{\sigma}, u_{\tau}, g_{\tau}$, and $\gamma$ so that the $\tau$ spins remain gapped, deep in their paramagnetic phase, and we tune a single parameter $g_{\sigma}$ to bring the $\sigma$ spins to criticality. Using exact diagonalization, we identify the location of the new critical point by studying the finitesize crossing of the gap of the system [see phase diagram in Fig. 4(a)]. We have verified that $H_{\mathrm{gSPT}}^{\prime}$ has gapless edge modes and anomalous magnetization for the parameter ranges $0 \leq u_{\sigma}, g_{\tau}, u_{\tau}, \delta \leq 0.2$ and $0 \leq \gamma \leq 1$.
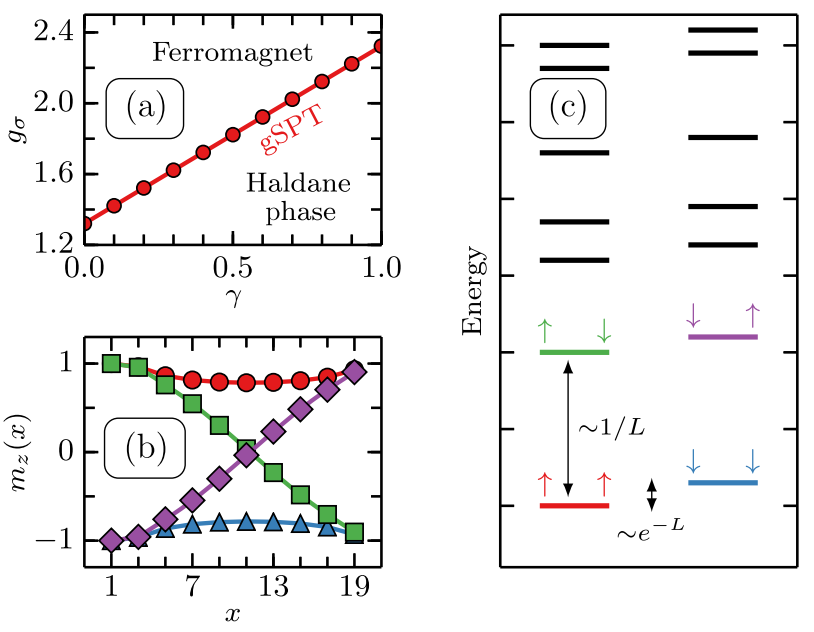

FIG. 4. (a) Phase diagram showing a gSPT line separating the Haldane and ferromagnetic phases obtained from ED on 12 and 16 sites with $\Delta_{\tau}=10, g_{\tau}=u_{\sigma}=0.1, u_{\sigma}=\delta=0.2$. (b) Magnetization profiles of the lowest four eigenstates via ED on 20 sites with the same parameters as (a), but fixing $\gamma=0.1$, which implies $g_{\sigma}=g_{\sigma}^{c} \approx 1.421$ at the $\mathrm{gSPT}$ point. To break the symmetry, a small magnetic field $\sim \mathrm{e}^{-L}$ in the $z$ direction is applied. (c) Cartoon spectrum of $H_{\mathrm{gSPT}}^{\prime}$. Colors of states correspond to magnetization profiles.
Away from the exactly solvable limit described in the previous section, the exact degeneracy of the ground state is lifted by quantum fluctuations. There are two nearly degenerate ground states, which correspond to cat-state superpositions $\left|+{ }_{1}+{ }_{L}\right\rangle \pm\left|--_{1}-{ }_{L}\right\rangle$ of the edges modes. The splitting between these two cat states with lowest energy remains generically protected by the gap of the $\tau$ spins and is exponentially small in system size, well below the finite-size CFT gap that scales as $\sim 1 / L$. The first excited states are also cat states corresponding to the configurations $\left|+_{1}-{ }_{L}\right\rangle,\left|--_{1}+_{L}\right\rangle$ of the edge modes. They are power-law split from the two ground states because the anti-aligned edge modes induce a change of boundary condition [Fig. 4(c)]. In the CFT language, this corresponds to the insertion of a boundary condition changing operators $[77,78]$, which leads to a finite-size gap $\pi v_{F} / 2 L$ for a system of size $L$ [79], with $v_{F}$ the Fermi velocity. Figure 4(b) shows the anomalous magnetization of the low-lying eigenstates for nontrivial parameter values, consistent with the above picture.

In conclusion, this system provides an example of a $1+1 d$ gapless SPT as a decorated critical Ising model. We showed that the anomalous edge properties of $H_{\mathrm{gSPT}}$ are robust and do not require any additional fine-tuning beyond making the $\sigma$ spins critical. This gSPT state can also be interpreted as a quantum critical point between a nontrivial SPT and a ferromagnet, although we emphasize again that our general construction also applies to gapless phases, including Luttinger liquids in $1 d$ (see below). The presence of exotic edge properties at this transition stands in contrast to previous works on transitions between trivial and nontrivial SPTs [80-83]. This should admit straightforward generalizations to Potts models and parafermions in the case of a $\mathbb{Z}_{N} \times \mathbb{Z}_{N}$ symmetry.

Numerically, the anomalous edge magnetization even appears to survive disorder. Because of the unitary twist relating $H_{\mathrm{gTrivial}}$ and $H_{\mathrm{gSPT}}$, the stability of the gSPT critical point against disorder is determined by the Harris criterion for the gTrivial system (disorder is irrelevant if the correlation length exponent satisfies $\nu \geq 2 / d$ ) and by the gap of the $\tau$ spins. Therefore, gSPTs should be as stable against disorder as their gTrivial counterparts before applying the unitary twist. Moreover, even if disorder is relevant, we expect that disordered examples of gSPT systems could be uncovered by studying the boundary physics of twisted infinite randomness critical points [84]. This could lead to "topological" random singlet phases both at zero temperature [84,85] and in the context of many-body localization [86-88]. Furthermore, the possible presence of a strong zero mode [89-91] in such models should be investigated.

\section{C. $U(1) \times \mathbb{Z}_{2} \times \mathbb{Z}_{2}$ gSPT phase in $1 d$}

The presence of a quantum critical point in the preceding example is a special case; our construction can be applied 

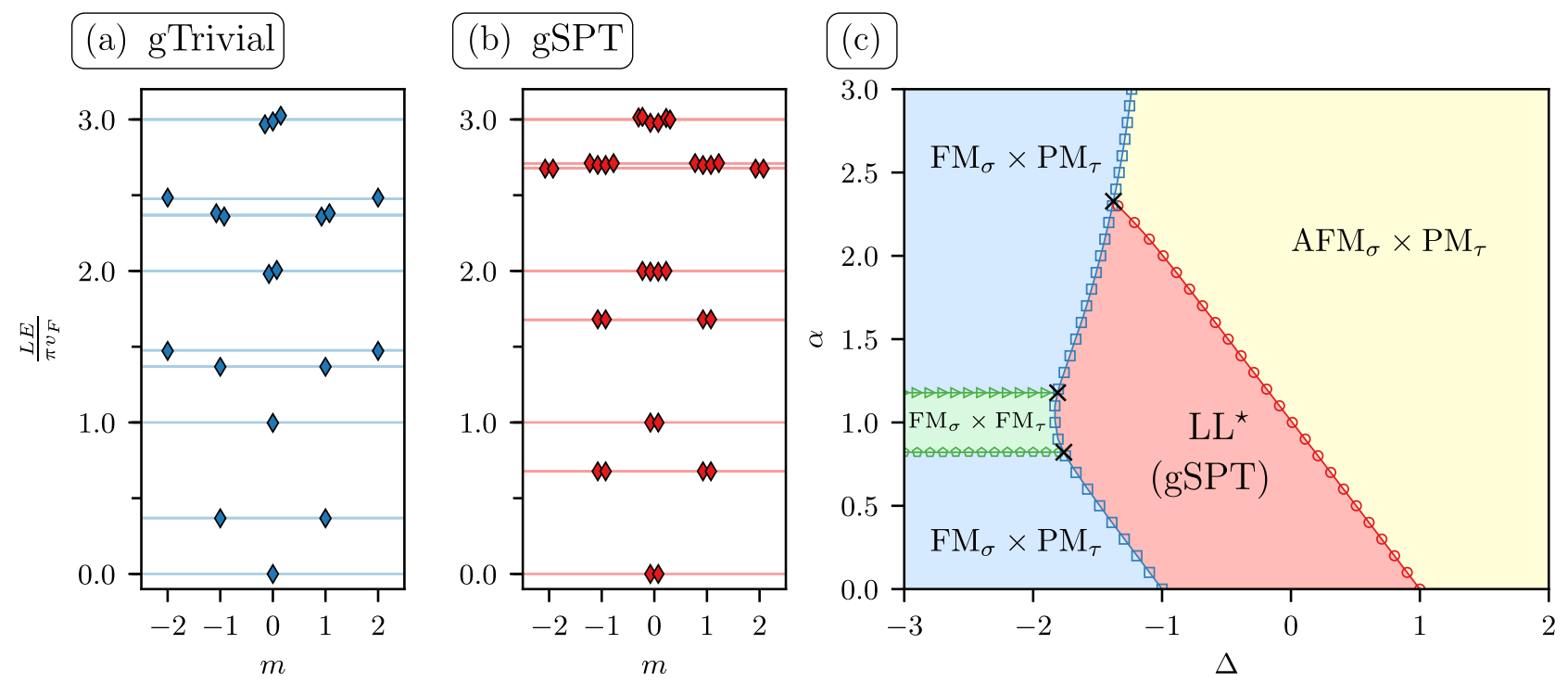

FIG. 5. (a) Spectrum of $H_{\mathrm{gTrivial}}^{\mathrm{LL}}$. (b) Spectrum of the gSPT $H_{\mathrm{gSPT}}^{\mathrm{LL} *}$. For both cases, spectra are normalized to be able to read off CFT operator dimensions. The conformal blocks are labeled by the magnetic charge sector $m$ and spaced horizontally, and small horizontal spacings show degenerate eigenvalues (up to exponential splitting). One can see that the states in the gSPT case are all doubly degenerate, because of the edge modes, but also that operator dimensions have changed relative to the gTrivial case. The numerical spectra were computed via DMRG [92] on up to 32 sites with finite-size scaling, and the solid lines correspond to the exponents expected from boundary CFT using $\Delta_{\text {eff }}=-\cos \pi g$ [93]. To improve convergence, the gap on the paramagnetic sector was increased from one to ten. (c) The phase diagram of $H_{\mathrm{gSPT}}^{\mathrm{LL} *}$, as computed via DMRG [92]. Each line denotes a different eigenvalue crossing, which accompanies a phase transition, and black crosses denote multicritical points. The Hamiltonian parameters used are $\Delta=-0.5, \alpha=0.1$, $g_{\tau}=0.3$, and $u_{\tau}=0.1$ for (a), (b), and (c).

not only to gapless points but equally well to lines or phases. To emphasize the generality of our construction, we now present a gapless SPT phase in $1 d$.

We start from a ("gTrivial") gapless phase in $1 d-\mathrm{a}$ Luttinger liquid [94]. The systematic nature of our construction allows us to closely follow the $\mathbb{Z}_{2} \times \mathbb{Z}_{2}$ example above, but we enforce an additional $U(1)$ symmetry on the gapless $\sigma$ spins in order to lock them into a Luttinger-liquid phase. We start from the Hamiltonian

$$
\begin{aligned}
H_{\mathrm{gTrivial}}^{\mathrm{LL}}= & \sum_{i} \sigma_{i}^{x} \sigma_{i+1}^{x}+\sigma_{i}^{z} \sigma_{i+1}^{z}+\Delta \sigma_{i}^{y} \sigma_{i+1}^{y} \\
& -\sum_{i} \tau_{i-\frac{1}{2}}^{x}+g_{\tau} \tau_{i-\frac{1}{2}}^{z} \tau_{i+\frac{1}{2}}^{z}+u_{\tau} \tau_{i-\frac{1}{2}}^{x} \tau_{i+\frac{1}{2}}^{x} \\
& +\sum_{i} \alpha \sigma_{i}^{y} \tau_{i+\frac{1}{2}}^{x} \sigma_{i+1}^{y},
\end{aligned}
$$

which describes gapless $\sigma$ spins ( $X X Z$ model) coupled through the $\alpha$ term to gapped Ising $\tau$ spins deep in their paramagnetic phase $\left(u_{\tau}\right.$ and $g_{\tau}$ are small). (Note that in contrast to the usual convention for the $X X Z$ spin chain, $\Delta$ adjusts the magnitude of the $\sigma^{y} \sigma^{y}$ interaction to make the symmetries more convenient.) This case has a $U(1) \rtimes \mathbb{Z}_{2}^{(\sigma)} \times$ $\mathbb{Z}_{2}^{(\tau)}$ global symmetry, generated by $U_{\theta}=\prod_{i} e^{i \theta \sigma_{i}^{y}}, \mathcal{C}_{\sigma}=$ $\prod_{i} \sigma_{i}^{x}$, and $\mathcal{C}_{\tau}=\prod_{i} \tau_{i+1 / 2}^{x}$, respectively. Assuming that $\alpha$ is small, the gapped $\tau$ spins can be integrated out to renormalize the anisotropy parameter $\Delta_{\text {eff }}=\Delta-\alpha\left\langle\tau^{x}\right\rangle$. The resulting $\sigma$ spins are gapless for $-1<\Delta_{\text {eff }} \leq 1$ and form, at low energies, a (single-channel) Luttinger-liquid phase with effective Lagrangian

$$
\mathcal{L}=\frac{g}{4 \pi}\left(\partial_{\mu} \phi\right)^{2},
$$

with $\Delta_{\text {eff }}=-\cos \pi g$, and $\phi$ a compact boson with unit compactification radius. (We set the Fermi velocity to $v_{F}=1$ for simplicity.)

Upon applying the unitary twist (3) and following the same steps as above, one can readily show that the twisted Hamiltonian $H_{\mathrm{gSPT}}^{\mathrm{LL}}=U_{1 d} H_{\mathrm{gTrivial}}^{\mathrm{LL}} U_{1 d}$ has edge modes in the limit $\alpha=g_{\tau}=u_{\tau}=0$ [95]. These topological edge modes are robust and persist away from this special limit as long as the gap of the $\tau$ spins does not close. Similarly to the $\mathbb{Z}_{2} \times \mathbb{Z}_{2}$ example above, the edge modes can be thought of as inducing a spontaneous edge magnetization along the $z$ direction, which in turn induces a change of (conformally invariant) boundary conditions [96]. Using standard bosonization techniques, the edge modes can be seen to lead to a doubly degenerate spectrum of boundary critical exponents that can be obtained from the gTrivial case through the substitution $g \rightarrow(1 / 4 g)$ [93]. (Note that this is in sharp contrast with the $\mathbb{Z}_{2} \times \mathbb{Z}_{2}$ gSPT discussed above, where the edge modes only led to degeneracies and did not modify the value of the critical exponents.) We have checked these predictions and the robustness of this 
topological Luttinger-liquid (gSPT) phase using exact diagonalization and DMRG calculations [93] (See Fig. 5).

We emphasize that, contrary to other examples of topological Luttinger liquids previously discussed in the literature [52,57], our construction does not rely on the spin-charge separation property of Luttinger liquids. Instead, our decorated domain-wall construction provides us with a systematic way of generating strongly interacting gapless SPT phases while making their topological nature transparent in clear analogy with gapped SPT systems.

\section{TWO DIMENSIONS}

To showcase the range of our general construction, our second example is a more involved system in $2 d$. However, the construction is parallel to the last section. We first define the model and then proceed to analyze its behavior in subsequent sections.

This example has a $G_{\sigma} \times G_{\tau}=\mathbb{Z}_{2} \times\left(\mathbb{Z}_{2} \times \mathbb{Z}_{2}\right)$ symmetry, where the domain walls of $G_{\sigma}=\mathbb{Z}_{2}$ will be decorated with (gapped) one-dimensional SPT states protected by $G_{\tau}=\mathbb{Z}_{2} \times \mathbb{Z}_{2}$. Let $A$ be a lattice whose sites host $\sigma$ spins, with symmetry $\mathcal{C}_{A}=\prod_{a \in A} \sigma_{a}^{x}$. The $\tau$ spins live on the sites of the dual (face-centered) lattice of $A$, called $A^{\star}$, which we assume to be bipartite so that $A^{\star}=B \cup C$ with symmetries given by $\mathcal{C}_{B}=\prod_{b \in B} \tau_{b}^{x}$ and $\mathcal{C}_{C}=\prod_{c \in C} \tau_{c}^{x}$. We further assume a symmetry exchanging $B$ and $C$. This can be realized either on triangular or Union Jack lattices, as shown in Fig. 2. A "trivial" paramagnetic state can be obtained as an equal-weight superposition of all classical configurations of spins, with parent Hamiltonian $H_{\text {Trivial }}=$ $-\sum_{a \in A} \sigma_{a}^{x}-\sum_{a^{\star} \in A^{\star}} \tau_{a^{\star}}^{x}$ and ground-state wave function

$$
\left|\Psi_{\text {Trivial }}\right\rangle=\sum_{\left\{\sigma^{z}\right\},\left\{\tau^{z}\right\}}\left|\sigma^{z}, \tau^{z}\right\rangle .
$$

Following the well-known construction [30,69], a parent Hamiltonian for a nontrivial $\mathbb{Z}_{2}^{3}$ SPT is given by $H_{\text {SPT }}=U_{2 d} H_{\text {Trivial }} U_{2 d}$, where $U_{2 d}=\prod_{\Delta_{i j k}} \mathrm{CCZ}_{i j k}$ is a local unitary operator that applies a three-qubit operator on each triangle of three neighboring $\mathrm{ABC}$ sites (see Fig. 2). This control-control- $Z$ operator gives a -1 for three down spins and +1 otherwise: $\mathrm{CCZ}_{i j k}=(-1)^{\delta_{\downarrow \downarrow \downarrow}}$. One can check that, for each edge $\langle j k\rangle$ of $A^{\star}$ that hosts a $\sigma$ domain wall, $U_{2 d}$ applies the two-qubit control- $Z$ operator $\mathrm{CZ}_{j k}$ on the $\tau$ spins $j$ and $k$. This unitary therefore applies $U_{1 d}$ to the $\tau$ spins living on each domain wall of the $\sigma$ spins, thereby decorating them with a $1 d$ SPT chain protected by a $\mathbb{Z}_{2} \times \mathbb{Z}_{2}$ symmetry. Explicitly, we have

$$
\left|\Psi_{\mathrm{SPT}}\right\rangle=U_{2 d}\left|\Psi_{\text {Trivial }}\right\rangle=\sum_{\left\{\sigma^{z}\right\},\left\{\tau^{z}\right\}} e^{i \theta_{2 d}\left(\sigma^{z}, \tau^{z}\right)}\left|\sigma^{z}, \tau^{z}\right\rangle,
$$

where the $\sigma$ and $\tau$ spins are now coupled through the phase factor $e^{i \theta_{2 d}\left(\sigma^{z}, \tau^{z}\right)}$, which takes care of the domain-wall decoration:

$$
e^{i \theta_{2 d}}=\prod_{\{\mathrm{dw}\}} e^{i \theta_{1 d}\left(\tau_{\mathrm{dw}}\right)} .
$$

Here, the product is over the domain walls of $\sigma^{z}$, denoted by $\{\mathrm{dw}\}$, and $e^{i \theta_{1 d}\left(\tau_{\mathrm{dw}}\right)}$ is defined in the previous section and applied to the $\tau$ spins living on a given domain wall.

For a region with an edge of $B$ and $C$ sites, $U_{2 d}$ does not modify the $G_{\tau}$ symmetry generators $\mathcal{C}_{B}$ and $\mathcal{C}_{C}$, but it does lead to additional boundary terms in $U_{2 d} \mathcal{C}_{A} U_{2 d}=$ $\prod_{a \in A} \sigma_{a}^{x} \prod_{\partial A^{*}} \mathrm{CZ}$, where $\mathrm{CZ}$ is a control- $Z$ gate giving a -1 factor if two successive $B$ and $C$ boundary spins are down. We can write down the edge theory of this $\mathbb{Z}_{2}^{3}$ SPT following Levin and Gu [28] by including all terms allowed by the symmetries, such as $\tau_{B, i-1}^{z} \tau_{C, i}^{x} \tau_{B, i+1}^{z}+\tau_{C, i}^{x}$, $\tau_{B, i-1}^{z} \tau_{B, i+1}^{z}$, and $B \leftrightarrow C$ permutations.

Using standard duality arguments, the edge theory can be thought of as two coupled Ising models tuned to their self-dual critical points (also known as the Ashkin-Teller model [97]). After bosonization, the edge excitations can be described by a Luttinger liquid [94] with central charge $c=1$ at the electromagnetic self-dual point

$$
\mathcal{L}_{\mathrm{SPT}}^{\text {edge }}=\frac{1}{4 \pi}\left(\partial_{\mu} \phi\right)^{2}-\lambda(\cos 2 \phi+\cos 2 \theta),
$$

where $\phi, \theta$ are compact conjugate bosonic fields with unit compactification radius. The edge is protected by the symmetries $\phi \rightarrow \pm \phi+\pi, \theta \rightarrow \pm \theta+\pi$, and $\phi \leftrightarrow \theta$ (note that the last symmetry is generated by the symmetry exchanging $B$ and $C$ spins). The vertex operators $\cos 2 \phi$ and $\cos 2 \theta$ correspond to products of the energy operators of the two Ising models. They are marginal perturbations that can be absorbed by renormalizing the Luttinger parameter and the sound velocity [98].

\section{A. gSPT wave function}

Now, we tune the $\sigma$ spins to criticality by imposing the constraint that the domain walls of the $\sigma^{z}$ spins must be fully packed loops (FPL) $[99,100]$. On the triangular lattice, this corresponds to a natural physical constraint: The allowed $\sigma^{z}$ states are the maximally antiferromagnetic ones, which, because of frustration, are known to have extensive degeneracy and power-law correlations [101]. On the square lattice, the FPL constraint is equivalent to the ice rule of the six-vertex model [102]. For concreteness, we focus on the triangular lattice, for which the fully packed loops live on the dual honeycomb lattice. For a given site $a \in A$, let $P_{a}$ be the projector onto allowed configurations (i.e., configurations that respect the constraint for the six triangles surrounding $a$ ), and let $P_{a}^{\prime}$ be the projector onto allowed configurations for which $a$ is "resonant" (i.e., configurations that would still respect the constraint after flipping $\sigma_{a}^{z}$ ). Since $P_{a}$ and $P_{a}^{\prime}$ are only functions of the $\sigma^{z}$ operators on $a$ and its neighbors (on the $A$ lattice), they are local operators. Then, the gTrivial Hamiltonian is (still with $G_{\sigma} \times G_{\tau}$ symmetry) 
$H_{\mathrm{gTrivial}}=\sum_{a \in A} \Lambda\left(1-P_{a}\right)+\sum_{a \in A} P_{a}^{\prime}\left(1-\sigma_{a}^{x}\right) P_{a}^{\prime}-\sum_{a^{\star} \in A^{\star}} \tau_{a^{\star}}^{x}$,

where $\Lambda \rightarrow \infty$ is an energy cost to penalize configurations that do not respect the constraint. To find the exact ground state, note that the $\tau$ spins are completely decoupled from the $\sigma$ spins. For the $\sigma$ d.o.f., we can follow the standard argument from Rokhsar and Kivelson [103]. The $\sigma$ part of $H_{\text {gTrivial }}$ is a sum of projectors and is therefore positive semi-definite. Thus, the (unnormalized) state

$$
\left|\Psi_{\text {gTrivial }}\right\rangle=\sum_{\left\{\sigma^{z}\right\},\left\{\tau^{z}\right\}}\left|\overline{\sigma^{z}}, \tau^{z}\right\rangle,
$$

an equal-weight superposition over all $\sigma^{z}$ states that satisfy the constraint (denoted $\overline{\left\{\sigma^{z}\right\}}$ ) times a paramagnetic state for the $\tau$ spins, has zero energy under the $\sigma$ part of $H_{\text {gTrivial }}$ and is hence an exact ground state. Equal-time $\sigma^{z}$-correlation functions in the ground state are described by correlation functions in the $2 d$ FPL model with loop fugacity $n=1[99,100]$ or, equivalently, by correlation functions in the zero-temperature, triangular-lattice, Ising antiferromagnet [101].

Using standard mappings onto dimers and height models $[99,100]$, the continuum limit of the $2 d$ FPL model can be identified as a $c=1$ compact boson CFT $\mathcal{L}=(g / 4 \pi)$ $(\nabla \varphi)^{2}-\gamma \cos 3 \varphi$, with $g=\frac{1}{2}$ and $\varphi \equiv \varphi+2 \pi$, so the perturbation $\cos 3 \varphi$ has scaling dimension $\Delta=9$ and is irrelevant. Following Refs. [104-106], we quantize this theory to identify the $2+1 d$ effective field theory describing the low-energy physics of $H_{\text {gTrivial }}$ as the $z=2$ quantum Lifshitz model (QLM) with (Euclidian) Lagrangian density

$\mathcal{L}_{\mathrm{QLM}}^{\text {bulk }}=\frac{1}{2}\left[\left(\partial_{\tau} \varphi\right)^{2}+k(\nabla \varphi)^{2}+\kappa^{2}\left(\nabla^{2} \varphi\right)^{2}\right]-\gamma \cos 3 \varphi$

tuned to $k=0$ with $\kappa=1 /(8 \pi)$ to reproduce the equaltime antiferromagnetic spin correlations on the triangular lattice. This constitutes an effective field theory for the gTrivial order on a closed manifold and is manifestly gapless. Equivalently, one can also think of this quantum critical point in terms of a dual $U(1)$ gauge theory with a quadratic photon mode [106].

The stability of this quantum critical point has been studied in various contexts $[105,107,108]$ and depends on crystalline symmetries, with the important relevant perturbations in our case being magnetic operators breaking the FPL constraint and $k(\nabla \varphi)^{2}$, which makes $\cos 3 \varphi$ relevant and opens up a gap [this can be equivalently interpreted as the instability of the deconfined phase of $U(1)$ gauge theories in $2+1 d$ [109].] In the following, we assume the bulk is tuned to this quantum Lifshitz critical point. The $\mathbb{Z}_{2}^{3}$ symmetry discussed above acts trivially on $\varphi$, but the theory (19) has additional crystalline symmetries corresponding to threefold rotations $\varphi \rightarrow \varphi+2 \pi / 3$ and inversion $\varphi \rightarrow-\varphi$. A similar field theory can be obtained on the square lattice [110].

An example of $2+1 d$ gapless SPT order is now obtained by decorating $H_{\text {gTrivial }}$,

$$
H_{\mathrm{gSPT}}=U_{2 d} H_{\mathrm{gTrivial}} U_{2 d}
$$

Its ground state is simply

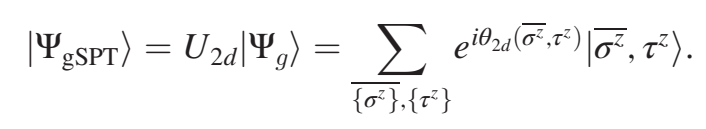

We now argue - crucially - that the critical wave function $\left|\Psi_{\mathrm{gSPT}}\right\rangle$ has an extra gapless edge mode compared to $\left|\Psi_{\text {gTrivial }}\right\rangle$ and that this edge mode is protected. This behavior is a hallmark of SPT order and must be treated with care in this gapless context. We therefore present three independent arguments for it: (1) effective field theory and boundary renormalization group (RG), (2) bulk-boundary correspondence, and (3) entanglement spectrum calculations with numerics. Each argument separately confirms a gapless $c=1$ edge in the gTrivial case and a gapless $c=2$ edge in the gSPT case.

\section{B. Edge field theory}

We first consider the edge modes of the (topologically trivial) gapless state $\left|\Psi_{\text {gTrivial }}\right\rangle$, Eq. (18), for which Eq. (19) describes the bulk behavior of the $\sigma$ spins. Because the boundary conditions for the spins are free, we consider Neumann boundary conditions for the field $\varphi$. [Note that Dirichlet boundary conditions $\left.\varphi\right|_{\partial}=0$ for the QLM are RG unstable and flow to Neumann, as the normal derivative boundary perturbation $\left.\left(\partial_{n} \varphi\right)^{2}\right|_{\partial}$ has scaling dimension $\Delta=2<z+1=3$ and is therefore relevant.] However, it is important that even though the relativistic $z=1$ term in Eq. (19) is tuned to $k=0$, such quadratic terms have no reason to be set to zero at the edge without additional finetuning. At the boundary, one should therefore add a lateral derivative boundary term $V \sim \delta^{2} \int_{\partial} d \tau d x_{\|}\left(\partial_{x_{\|}} \varphi\right)^{2}$ to the action. Here, $x_{\|}$is the coordinate along the edge and $\delta$ is a nonuniversal parameter. The boundary perturbation $\left(\partial_{x_{\|}} \varphi\right)^{2}$ is relevant, and we conjecture that, in the IR, it endows the edge with $z=1$ dynamics (forgetting the slower $z=2$ bulk dynamics). This result leads to the effective low-energy action for the edge theory of the QLM,

$$
S_{\mathrm{QLM}}^{\mathrm{edge}}=\frac{1}{2} \int d \tau d x_{\|}\left[\left(\partial_{\tau} \varphi\right)^{2}+\delta^{2}\left(\partial_{x_{\|}} \varphi\right)^{2}\right]+\cdots,
$$


where the dots represent less RG-relevant terms. This is the action of a $1+1 d$ compact boson CFT with central charge $c=1$. We emphasize that the effective Luttinger parameter is nonuniversal and set by the value of $\delta$, which, depending on microscopic parameters, could lead to a gapped edge because of the cosine terms dropped in Eq. (22). The existence of this edge has nothing to do with the SPT, and indeed, the symmetries $\left(\mathcal{C}_{A}, \mathcal{C}_{B}, \mathcal{C}_{C}\right)$ act trivially on $\varphi$. The $\sigma$ spins therefore have a $z=2$ bulk with diffusive dynamics and can have a $z=1$ edge with ballistic dynamics. To our knowledge, edge modes for gapless systems have very rarely been discussed in the literature [111-115]. The presence of an edge is confirmed numerically below.

We now turn to the edge theory of the gSPT wave function (21). Upon integrating out the gapped $\tau$ d.o.f., we expect the bulk low-energy theory to be described by Eq. (19), with $k=0$, where the boundary actions (16) and (22) (both with nonuniversal Luttinger parameters) are coupled through all symmetry-allowed perturbations. The essential point is that the SPT $\mathbb{Z}_{2}^{3}$ symmetry acts trivially on $\varphi$, so perturbations such as $\cos (\varphi-\phi)$ that could generically gap out the edge are not allowed by symmetry. Intuitively, coupling the two edge theories does not increase the number of symmetry-allowed perturbations since they are protected by distinct symmetries. There is therefore a finite range of Luttinger parameters for which the edge is gapless with central charge $c=2$. Moreover, the SPT part of the edge, described by Eq. (16), is symmetry protected as it can only be gapped out by condensing $\phi$ or $\theta$, thereby spontaneously breaking the $\mathbb{Z}_{2}^{3}$ symmetry. A related mechanism for noninteracting gapless topological superconductors and insulators has been discussed in Ref. [115].

Investigating to what extent these edge modes leak into the gapless bulk is a complicated task. In analogy with the $1 d$ case, at least the $\tau$ component of the edge should be exponentially localized despite the critical bulk. Even though the domain walls themselves are critical, the $1+1 d$ SPT chains that live on them are still gapped. Therefore, when a domain wall ends at a boundary, there is a free spin-1/2 living at the end point (see Fig. 1) whose only way to move towards the bulk is along the domain wall, which is forbidden by the $1+1 d$ SPT gap. We thus expect the free edge spins to be exponentially localized, where the localization length is given by the gap on the $1+1 d$ SPT chains.

\section{Bulk-boundary correspondence}

The bulk-boundary correspondence for fractional quantum Hall (FQH) states [116-119] is a powerful technique whereby a $\mathrm{FQH}$ wave function is written as a correlator in a CFT. When it is unitary, the CFT also gives the edge and entanglement spectra [120-122]. This correspondence was recently extended to SPT wave functions [123] (see also Ref. [124]), and we build on this result in this section.

Starting from a SPT wave function, a convenient way of identifying its underlying CFT is to compute the "strange correlator theory" $Z_{\Psi_{\mathrm{SPT}}} \equiv\left\langle\Psi_{\text {Trivial }} \mid \Psi_{\mathrm{SPT}}\right\rangle$ [125]. The idea behind this theory is that correlators of the type $\left\langle\Psi_{\text {Trivial }}\left|\mathcal{O}_{1} \mathcal{O}_{2}\right| \Psi_{\text {SPT }}\right\rangle$ measure observables on an edge in imaginary time between a trivial and a nontrivial SPT and can therefore probe the edge physics. To complete the analogy with the FQH bulk-boundary correspondence, it was shown in Ref. [123] that $\left|\Psi_{\mathrm{SPT}}\right\rangle$ can be written in terms of correlators in the $Z_{\Psi_{\text {SPT }}}$ CFT.

We now calculate $Z_{\Psi}$ for both the gapped and gapless SPT. Let us first briefly review the gapped case $[123,125]$. We focus on the triangular lattice, but the results generalize straightforwardly to the Union Jack lattice. Starting from Eqs. (14) and (15), one can use the fact that $e^{i \theta_{2 d}}$ factors over domain walls to analytically sum the $\tau$ d.o.f. over each domain wall separately. This yields [123]

$$
Z_{\Psi_{\mathrm{SPT}}}=\sum_{\left\{\sigma^{z}\right\},\left\{\tau^{z}\right\}} e^{i \theta_{2 d}\left(\sigma^{z}, \tau^{z}\right)} \propto \sum_{\{\mathrm{dw}\}} n^{N[\mathrm{dw}]} x^{L[\mathrm{dw}]},
$$

with $L[\mathrm{dw}]$ the total length of domain walls, $N[\mathrm{dw}]$ the total number of domain walls, $x^{-1}=\sqrt{2}$, and $n=2$. Since domain walls form closed, nonintersecting loops, this can be identified as a dense (but not fully packed) loop model on the honeycomb lattice with loop fugacity $n=2$ and loop tension $x^{-1}=\sqrt{2}$ [126]. For these parameters, this loop model is exactly solvable and is given by the $S U(2)_{1}$ CFT with central charge $c=1$ [126], in agreement with the edge field theory given in Eq. (16).

The strange correlator theory for the gapless case can be calculated analogously by restricting to fully packed loop configurations $\overline{\{\mathrm{dw}\}}$. This leads to

$$
Z_{\Psi_{\mathrm{gSPT}}}=\sum_{\left\{\sigma^{2}\right\},\left\{\tau^{2}\right\}} e^{i \theta_{2 d}\left(\sigma^{2}, \tau^{2}\right)} \propto \sum_{\{\mathrm{dw}\}} n^{N[\overline{\mathrm{dw}}]},
$$

with $n=2$ again. This loop model is also known to give a CFT but with $c=2$ instead [100]. Hence, the bulk wave function of gSPT can be written as a correlator in a $c=2$ CFT, which is good evidence for a $c=2$ edge. It is remarkable that this imaginary-time edge picture holds for a nonrelativistic bulk theory with $z=2$. Notice that our analysis has provided us with a natural way of interpolating from the gapped SPT to the gapless SPT by tuning the loop tension $x^{-1}$ from $\sqrt{2}$ to zero. In the following section, we give further evidence by showing that the entanglement spectrum is given by a $c=2$ theory as well.

\section{Entanglement spectrum}

A useful property of entanglement cuts in systems that obey the area law for entanglement entropy $[127,128]$ is that the corresponding entanglement Hamiltonian can be interpreted as an "edge" Hamiltonian [120]. This correspondence between entanglement and edge Hamiltonians has been shown rigorously in certain cases [122] and has 


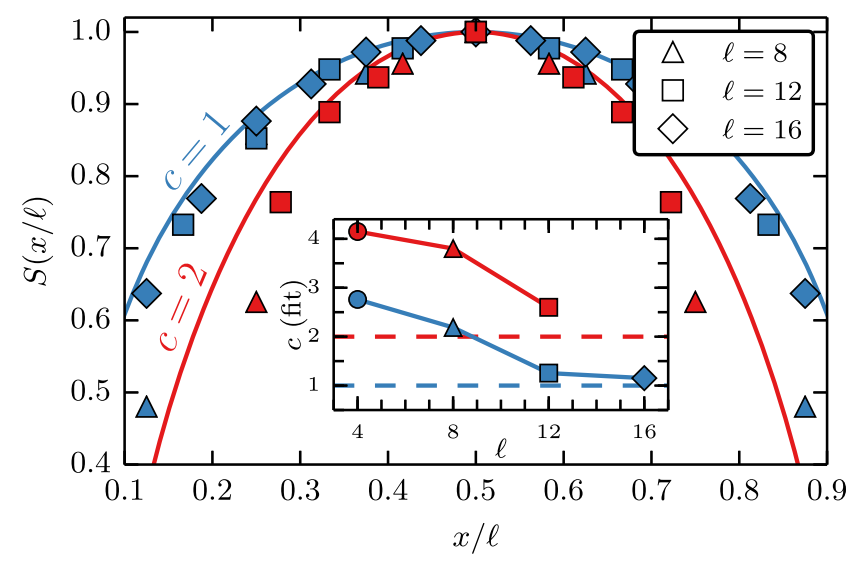

FIG. 6. Entanglement entropy of the $1+1 d$ ground state of the entanglement Hamiltonian $H_{\mathrm{E}}=-\log \rho$, with $\rho$ the reduced density matrix for a bipartite cut on an infinite cylinder of circumference $\ell$. Blue points are for $\left|\Psi_{\text {gTrivial }}\right\rangle$, and red points are for $\left|\Psi_{\mathrm{gSPT}}\right\rangle$. Data are offset so that $S(1 / 2)=1$.

been very useful in the numerical identification of various topological phases of matter. While so far this technique has been mostly used for systems that are gapped in the bulk, we emphasize that this is not an inherent limitation. As long as the area law is respected, it is always possible in practice to interpret the entanglement Hamiltonian as an edge Hamiltonian (see, for example, Ref. [112], where a gapless chiral spin liquid is shown to have an entanglement spectrum described by a CFT).

Consider $\left|\Psi_{\mathrm{gTrivial}}\right\rangle$ and $\left|\Psi_{\mathrm{gSPT}}\right\rangle$ on cylinders of circumference $\ell$ and infinite length. We make an entanglement cut transverse to the cylinder, which splits it into two semiinfinite regions, and we compute the reduced density matrix $\rho=e^{-H_{E}}$. As explained in the Appendix, using special properties of our exact ground state wave-functions, it is possible to show that they satisfy the area law [129], to find the exact Schmidt decomposition, and thence compute the entanglement spectrum by numerical exact diagonalization of a two-dimensional transfer matrix. The reduced density matrix has support only on the entanglement cut, so it naturally describes a $1 d$ system. Moreover, it has the form of a transfer matrix for a $2 d$ statistical model, so the quantum-classical mapping provides $H_{E}$ as a local operator.

We find that for both gTrivial and gSPT, the spectral gap of $H_{E}$ goes like $1 / \ell$, indicating the edges are indeed gapless (see the Appendix for details). To identify the CFT described by $H_{E}$ as a $1+1 d$ theory on a circle, we compute the entanglement entropy $S(x)$ of the ground state of $H_{E}$ for cuts of length $x$. We then apply the standard result of Cardy and Calabrese to extract the central charge $[130,131]$ :

$$
S(x)=\frac{c}{3} \ln \sin \frac{\pi x}{\ell} .
$$

Figure 6 shows $S(x)$ for the gTrivial and gSPT cases. As $\ell \rightarrow \infty$, this converges from below [132] to Eq. (25).
In the inset, the central charges are seen to converge to $c=1$ and $c=2$ for the gTrivial and gSPT orders, respectively.

We may thus conclude, having shown it by three independent and consistent methods, that the gapless trivial state has an edge mode with central charge $c=1$, while the gapless SPT case has $c=2$-recall, however, that only half of this edge is protected by the $\mathbb{Z}_{2}^{3}$ symmetry. These ballistic edge modes $(z=1)$ in a diffusive $(z=2)$ quantum critical system should have dramatic consequences for transport properties.

\section{CONCLUSIONS}

Gapless symmetry-protected order was proposed as a class of quantum matter. We provide a general construction for many gSPT systems by decorating the domain walls of gapless systems. To concretely understand gSPTs, we focused on two analytically solvable examples: a simple $1 d$ system that extends the Ising model and a gapless spin liquid in two dimensions. These examples demonstrate that gSPTs not only extend the crucial topological feature of SPTs - robust gapless edge modes-but also permit generalizations of tools developed for the gapped case, such as the bulk-boundary correspondence and the use of the entanglement spectrum as a probe of the edge. Both systems also exhibit exotic boundary behavior, including anomalous edge magnetization in the $1 d$ example and, for $2 d, z=1$ edge dynamics for a $z=2$ system. Both in $1 d$ and $2 d$, the gapless edge modes appear to be exponentially localized by the gap of the $\tau$ spins, even though they induce an algebraic disturbance for the $\sigma$ spins into the critical bulk.

These are by no means the only gSPTs. To wit, in the $1 d$ example, one could straightforwardly replace the Ising spins with parafermions or a Potts model; the $2 d$ example should permit a gapless topological state with relativistic Majorana edge modes using Majorana chains as decoration $[133,134]$, and it might be possible to find 3D gapless spin liquids where analytic control over the decoration is possible [135-137]. Two-dimensional gSPT states could also be realized in realistic strongly correlated electronic systems $[68,83]$.

More broadly, some of our examples can be interpreted as "twisted" quantum phase transitions between SPT and broken-symmetry phases, which are expected to be more generic than direct transitions from trivial to SPT phases [80-82]. Even if the bulk universality class of such twisted transitions is the same as for quantum critical points between trivial paramagnets and symmetry-broken phases (and hence described by conventional Ginzburg-Landau theory), our results indicate that twisted transitions differ from regular transitions in terms of surface criticality, in agreement with recent Monte Carlo results [64]. From a field theory perspective, trivial paramagnets and gapped SPTs can be understood as nonlinear sigma models in their gapped, disordered phase, the only difference being that the latter has a topological $\theta$ term with $\theta=2 \pi[34,70]$. 
It would be interesting to study the role of this $\theta$ term on the transition to symmetry-broken phases.

We also emphasize that our construction leads to gSPT states that are just as stable as the underlying gTrivial wave functions before applying the decoration. In particular, our construction yields stable gSPT phases by decorating Luttinger liquids in $1 d$ (see Sec. III C) or $U(1)$ gauge theories in 3D (left for future work). It would also be interesting to relate gSPTs to other gapless topological states of matter, including gapless fractionalized states $[65,138,139]$, in particular, by partially gauging the symmetries [28]. We leave these directions for future work, and we hope that gapless SPTs might provide a useful starting point to systematically study gapless topological matter.

\section{ACKNOWLEDGMENTS}

We thank S. Parameswaran, A. C. Potter, Z. Ringel, S. Simon, and B. Ware for insightful discussions, and Y.-M. Lu, A. Nahum, A. C. Potter, Z. Ringel, and Y.-Z. You for useful comments on the manuscript. We acknowledge support from the Emergent Phenomena in Quantum Systems initiative of the Gordon and Betty Moore Foundation (T.S.), NSF DMR-1507141 (D.P.), and the Department of Energy through the Quantum Materials program of LBNL (R. V.). R. V. also acknowledges support through the Supporting Open Access Research (SOAR) fund at UMass Amherst.

\section{APPENDIX: ENTANGLEMENT SPECTRUM ON THE CYLINDER}

This appendix computes the entanglement spectrum of the $2+1 d$ gapless states introduced in Sec. IV. Below, we explicitly calculate the reduced density matrix for both the "gapless trivial" and "gapless SPT" systems and show it may be written in terms of a transfer matrix for a gapless $1+1 d$ system, which we interpret as the edge theory. Using techniques of $1+1 d$ CFT [130,131], we demonstrate that this edge theory has $c=1$ for the gapless trivial case but $c=2$ for the gapless SPT case.

Consider a cylinder with a circumference of $\ell$ and infinite length (see Fig. 7). The analytic results below are general for any geometry, but for numerical convenience, we work on the (tilted) Union Jack lattice. The circumference $\ell$ is defined so that every column is composed of $\ell$ sites. Let us consider an entanglement cut transverse to the cylinder, which divides the cylinder into a left $(L)$ and right $(R)$ side. Figure 7 shows the geometry and sets notation.

With notation from Fig. 7, the gSPT wave function Eq. (21) can be written more explicitly as

$$
\begin{aligned}
\left|\Psi_{\mathrm{gSPT}}\right\rangle= & \frac{1}{\sqrt{Z}} \sum_{\{\sigma\},\{\tau\}} e^{i \theta(L)} e^{i \theta(R)} e^{i \theta\left(\partial_{L}, \partial_{R}\right)} \\
& \times\left|\sigma_{L}, \tau_{L}, \sigma_{\partial L}, \tau_{\partial L}\right\rangle \otimes\left|\sigma_{R}, \tau_{R}, \sigma_{\partial R}, \tau_{\partial R}\right\rangle,
\end{aligned}
$$

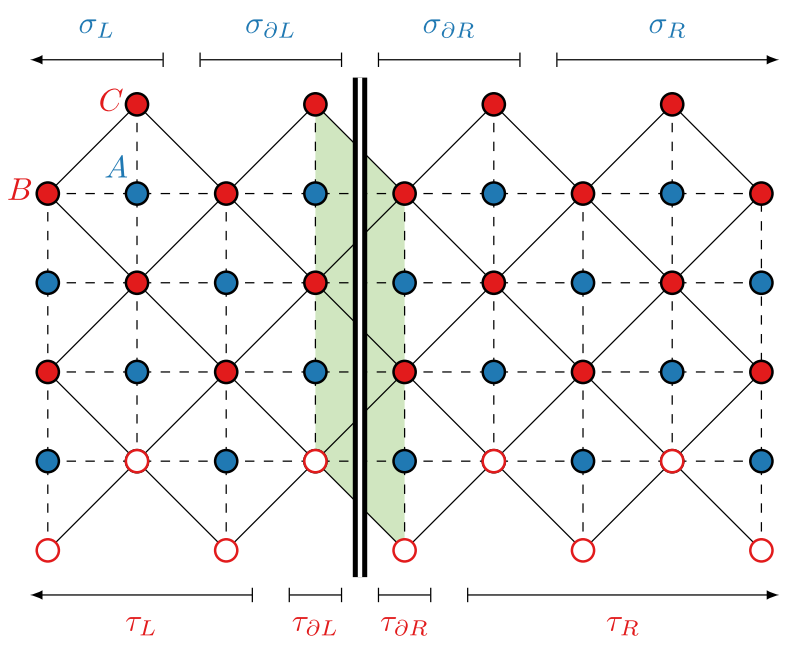

FIG. 7. The Union Jack lattice showing the $A$ sublattice (blue) and BC sublattices (red) on a cylinder. The empty sites on the bottom are identified with the full ones on top, giving a circumference $\ell=4$. The entanglement cut is denoted with a double line, and the triangles $\mathrm{ABC}$ that it breaks are highlighted in green. The top and bottom show the extent of the left, right, and boundary regions for each species of spins.

where the sum runs over all configurations of the $\sigma^{z}$ spins whose domain walls are FPL and over all $\tau^{z}$ spins. $Z$ factors into the partition function of the FPL model for $\sigma$ and a trivial normalization factor for $\tau$ :

$$
Z=\sum_{\{\sigma\},\{\tau\}} 1=2^{\# \tau} \sum_{\overline{\{\sigma\}}} 1
$$

with \# $\tau$ the number of $\tau$ spins. For a domain $D$, the phase factor $e^{i \theta(D)}$ gives a factor of -1 for each triangle strictly included in $D$ with three down spins. The triangles that cross the cut and contribute to $e^{i \theta(\partial L, \partial R)}$ are highlighted in green in Fig. 7.

Define wave functions on the left side for each possible choice of spin configurations at the left boundary (denoted $\partial L)$ by

$$
\begin{aligned}
& \left|\Psi_{\mathrm{gSPT}}^{L}\left[\sigma_{\partial L}, \tau_{\partial L}\right]\right\rangle
\end{aligned}
$$

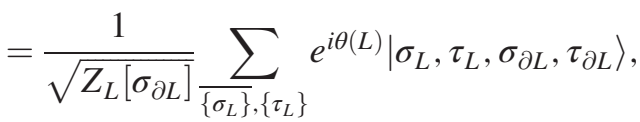

where $Z_{L}$ is the partition function on the left side, and it is independent of $\tau_{L}$. Define $\left|\Psi_{\mathrm{SPT}}^{R}\left[\sigma_{\partial R}, \tau_{\partial R}\right]\right\rangle$ analogously on the right side. In a dual picture, the domain walls of the $\sigma$ spins are isomorphic to configurations of the six-vertex model. This local constraint would allow an exact Schmidt decomposition following Ref. [140]. However, different cuts make physical sense with domain walls instead of spins. (Indeed, using the domain walls leads to a factorization of the density matrix as a product of the $\sigma$ and $\tau$ d.o.f.) 
Therefore, we emphasize that one must work with the actual spins. Conveniently, one may still use the local constraint on the $\sigma$ spins together with the zero correlation length of the $\tau$ spins to find an exact Schmidt decomposition.

We may rewrite the entire wave function as

$$
\begin{aligned}
\left|\Psi_{\mathrm{gSPT}}\right\rangle= & \sum_{\substack{\left\{\sigma_{\partial L}, \sigma_{\partial R}\right\} \\
\left\{\tau_{\partial L}, \tau_{\partial R}\right\}}}\left(\frac{Z_{L}\left[\sigma_{\partial L}\right] Z_{R}\left[\sigma_{\partial R}\right]}{Z}\right)^{1 / 2} e^{i \theta(\partial L, \partial R)} \\
& \times T_{\sigma_{\partial L}, \sigma_{\partial R}}\left|\Psi_{\mathrm{SPT}}^{L}\left[\sigma_{\partial L}, \tau_{\partial L}\right]\right\rangle \otimes\left|\Psi_{\mathrm{SPT}}^{R}\left[\sigma_{\partial R}, \tau_{\partial R}\right]\right\rangle,
\end{aligned}
$$

where the sum over $\sigma_{\partial L}$ and $\sigma_{\partial R}$ is now unconstrained. Here, $T$ is the transfer matrix for the fully packed loop model with loop fugacity one. Its role is to enforce the FPL constraint between the left and right sides. In the following, we use the orthogonality property

$$
\left\langle\Psi_{\mathrm{SPT}}^{L}\left[\sigma_{\partial L}^{\prime}, \tau_{\partial L}^{\prime}\right] \mid \Psi_{\mathrm{SPT}}^{L}\left[\sigma_{\partial L}, \tau_{\partial L}\right]\right\rangle=\delta_{\sigma_{\partial L}^{\prime}, \sigma_{\partial L}} \delta_{\tau_{\partial L}^{\prime}, \tau_{\partial L}}
$$

Starting from the density matrix $\rho=\left|\Psi_{\mathrm{gSPT}}\right\rangle\left\langle\Psi_{\mathrm{gSPT}}\right|$, we may use Eq. (A4) to immediately write the reduced density matrix on the left side:

$$
\left\langle\Psi_{\mathrm{SPT}}^{L}\left[\sigma_{\partial L}, \tau_{\partial L}\right]\left|\rho_{L}\right| \Psi_{\mathrm{SPT}}^{L}\left[\sigma_{\partial L}^{\prime}, \tau_{\partial L}^{\prime}\right]\right\rangle=\left(S S^{t}\right)_{\sigma_{\partial L} \tau_{\partial L}, \sigma_{\partial L}^{\prime} \tau_{\partial L}^{\prime}}
$$

where we used the above orthogonality property and where $S$ is a transfer matrix from the left to the right side,

$$
\begin{aligned}
S_{\sigma_{\partial L}, \tau_{\partial L}, \sigma_{\partial R}, \tau_{\partial R}}= & \left(\frac{Z_{L}\left[\sigma_{\partial L}\right] Z_{R}\left[\sigma_{\partial R}\right]}{Z}\right)^{1 / 2} \\
& \times T_{\sigma_{\partial L} \sigma_{\partial R}} e^{i \theta\left(\sigma_{\partial L}, \tau_{\partial L}, \sigma_{\partial R}, \tau_{\partial R}\right)} .
\end{aligned}
$$

The reduced density matrix manifestly depends only on the d.o.f. at the entanglement cut, whereas generically, it might depend on all the spins on the left side. If we define the entanglement Hamiltonian via $\rho_{L}=e^{-H_{E}}$, then $H_{E}$ describes a $1+1 d$ system on the boundary d.o.f. To compute the spectrum of $H_{E}$ on the cylinder, we use the fact that

$$
\begin{aligned}
Z_{L}\left[\sigma_{\partial L}\right] & =\lim _{N \rightarrow \infty} 2^{\# \tau} \sum_{\{\sigma\}}\left\langle\sigma\left|T^{N}\right| \sigma_{\partial L}\right\rangle \\
& =\lim _{N \rightarrow \infty} 2^{\# \tau} \sum_{\{\sigma\}}\langle\sigma \mid R\rangle \lambda^{N}\left\langle L \mid \sigma_{\partial L}\right\rangle,
\end{aligned}
$$

where, by the Perron-Frobenius theorem, $T^{N} \rightarrow|R\rangle \lambda^{N}\langle L|$, with $\lambda$ the largest eigenvalue of $T$, and $|R\rangle$ and $|L\rangle$ the corresponding right and left eigenvectors. The sum runs over all configurations of $\sigma^{z}$ on one column. This implies
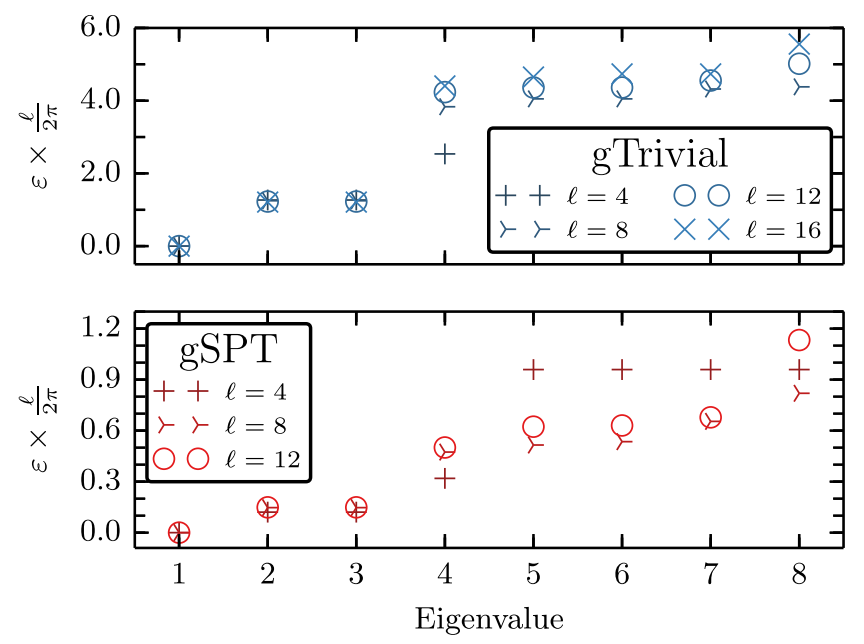

FIG. 8. Entanglement spectral gaps for the gTrivial and gSPT for entanglement cuts of the $2+1 d$ example on the cylinder of circumference $\ell$. The vertical axis is rescaled to be able to directly read off the operator dimensions of the excitations (up to a nonuniversal sound velocity). Only the first few excitations are well converged for this range of $\ell$.

$$
\begin{aligned}
& S_{\sigma_{\partial L}, \tau_{\partial L}, \sigma_{\partial R}, \tau_{\partial R}} \\
& \quad=\left(\frac{\left\langle L \mid \sigma_{\partial L}\right\rangle\left\langle\sigma_{\partial R} \mid R\right\rangle}{\langle L \mid R\rangle \lambda 2^{\ell}}\right)^{1 / 2} T_{\sigma_{\partial L} \sigma_{\partial R}} e^{i \theta\left(\sigma_{\partial L}, \tau_{\partial L}, \sigma_{\partial R}, \tau_{\partial R}\right)} .
\end{aligned}
$$

One can check that this is properly normalized: $\operatorname{Tr} \rho_{L}=$ $\operatorname{Tr} S S^{t}=1$.

We now employ exact diagonalization. At size $\ell, S$ is a $2^{3 \ell / 2} \times 2^{3 \ell / 2}$ matrix, making exact diagonalization practical for $\ell=4,8,12$. We restrict to the case where $\ell$ is a multiple of 4 in order to stay in the symmetric ground-state sector of the loop model. Since the $\tau$ part of the matrix is dense, larger sizes are impractical. However, in the gapless trivial case, we may discard the $\tau$ part and work on larger systems. For both the gapless trivial (where $e^{i \theta} \equiv 1$ ) and gapless SPT cases, the spectral gap for $H_{E}$ goes as $1 / \ell$, which indicates gaplessness with dynamical exponent $z=1$. This is shown in Fig. 8 .

By looking at the ground state of $H_{E}|0\rangle=\varepsilon_{0}|0\rangle$, we may determine the central charge of $H_{E}$ by making entanglement cuts in the $(1+1 d)$ edge system and comparing to the Cardy-Calabrese equation (25). For each $\ell$, a one-parameter fit to the Cardy-Calabrese result was performed to extract the central charge. Figure 6 shows that the central charge converges to $c=1$ in the gapless trivial case and $c=2$ in the gapless SPT case.

[1] A. Kitaev and J. Preskill, Topological Entanglement Entropy, Phys. Rev. Lett. 96, 110404 (2006).

[2] M. Levin and X.-G. Wen, Detecting Topological Order in a Ground State Wave Function, Phys. Rev. Lett. 96, 110405 (2006). 
[3] X. G. Wen, Quantum Field Theory of Many-Body Systems: From the Origin of Sound to an Origin of Light and Electrons, Oxford Graduate Texts (Oxford University Press, Oxford, 2004).

[4] X.-G. Wen, Quantum Orders and Symmetric Spin Liquids, Phys. Rev. B 65, 165113 (2002).

[5] J. Maciejko, X.-L. Qi, A. Karch, and S.-C. Zhang, Fractional Topological Insulators in Three Dimensions, Phys. Rev. Lett. 105, 246809 (2010).

[6] B. Swingle, M. Barkeshli, J. McGreevy, and T. Senthil, Correlated Topological Insulators and the Fractional Magnetoelectric Effect, Phys. Rev. B 83, 195139 (2011).

[7] M. Levin and A. Stern, Classification and Analysis of TwoDimensional Abelian Fractional Topological Insulators, Phys. Rev. B 86, 115131 (2012).

[8] A. Mesaros and Y. Ran, Classification of Symmetry Enriched Topological Phases with Exactly Solvable Models, Phys. Rev. B 87, 155115 (2013).

[9] Y.-M. Lu and A. Vishwanath, Classification and Properties of Symmetry-Enriched Topological Phases: Chern-Simons Approach with Applications to $Z_{2}$ Spin Liquids, Phys. Rev. B 93, 155121 (2016).

[10] L.-Y. Hung and X.-G. Wen, Quantized Topological Terms in Weak-Coupling Gauge Theories with a Global Symmetry and Their Connection to Symmetry-Enriched Topological Phases, Phys. Rev. B 87, 165107 (2013).

[11] C. L. Kane and E. J. Mele, Quantum Spin Hall Effect in Graphene, Phys. Rev. Lett. 95, 226801 (2005).

[12] B. A. Bernevig, T. L. Hughes, and S.-C. Zhang, Quantum Spin Hall Effect and Topological Phase Transition in HgTe Quantum Wells, Science 314, 1757 (2006).

[13] M. Konig, S. Wiedmann, C. Brune, A. Roth, H. Buhmann, L. W. Molenkamp, X.-L. Qi, and S.-C. Zhang, Quantum Spin Hall Insulator State in HgTe Quantum Wells, Science 318, 766 (2007).

[14] L. Fu, C. L. Kane, and E. J. Mele, Topological Insulators in Three Dimensions, Phys. Rev. Lett. 98, 106803 (2007).

[15] J.E. Moore and L. Balents, Topological Invariants of Time-Reversal-Invariant Band Structures, Phys. Rev. B 75, 121306 (2007).

[16] R. Roy, Topological Phases and the Quantum Spin Hall Effect in Three Dimensions, Phys. Rev. B 79, 195322 (2009).

[17] D. Hsieh, D. Qian, L. Wray, Y. Xia, Y. S. Hor, R. J. Cava, and M.Z. Hasan, A Topological Dirac Insulator in a Quantum Spin Hall Phase, Nature (London) 452, 970 (2008).

[18] M. Zahid Hasan and C. L. Kane, Colloquium: Topological Insulators, Rev. Mod. Phys. 82, 3045 (2010).

[19] B. Rasche, A. Isaeva, M. Ruck, S. Borisenko, V. Zabolotnyy, B. Büchner, K. Koepernik, C. Ortix, M. Richter, and J. van den Brink, Stacked Topological Insulator Built from Bismuth-Based Graphene Sheet Analogues, Nat. Mater. 12, 422 (2013).

[20] P. Dziawa, B. J. Kowalski, K. Dybko, R. Buczko, A. Szczerbakow, M. Szot, E. Łusakowska, T. Balasubramanian, B. M. Wojek, M. H. Berntsen, O. Tjernberg, and T. Story, Topological Crystalline Insulator States in $\mathrm{Pb}_{x-1} \mathrm{Sn}_{x} \mathrm{Se}$, Nat. Mater. 11, 1023 (2012).
[21] Z.-C. Gu and X.-G. Wen, Tensor-Entanglement-Filtering Renormalization Approach and Symmetry-Protected Topological Order, Phys. Rev. B 80, 155131 (2009).

[22] X. Chen, Z.-C. Gu, and X.-G. Wen, Complete Classification of One-Dimensional Gapped Quantum Phases in Interacting Spin Systems, Phys. Rev. B 84, 235128 (2011).

[23] A. M. Turner, F. Pollmann, and E. Berg, Topological Phases of One-Dimensional Fermions: An Entanglement Point of View, Phys. Rev. B 83, 075102 (2011).

[24] L. Fidkowski and A. Kitaev, Topological Phases of Fermions in One Dimension, Phys. Rev. B 83, 075103 (2011).

[25] X. Chen, Z.-X. Liu, and X.-G. Wen, Two-Dimensional Symmetry-Protected Topological Orders and Their Protected Gapless Edge Excitations, Phys. Rev. B 84, 235141 (2011).

[26] F. Pollmann, E. Berg, A. M. Turner, and M. Oshikawa, Symmetry Protection of Topological Phases in OneDimensional Quantum Spin Systems, Phys. Rev. B 85, 075125 (2012).

[27] Y.-M. Lu and A. Vishwanath, Theory and Classification of Interacting Integer Topological Phases in Two Dimensions: A Chern-Simons Approach, Phys. Rev. B 86, 125119 (2012).

[28] M. Levin and Z.-C. Gu, Braiding Statistics Approach to Symmetry-Protected Topological Phases, Phys. Rev. B 86, 115109 (2012).

[29] X. Chen, Z.-C. Gu, Z.-X. Liu, and X.-G. Wen, SymmetryProtected Topological Orders in Interacting Bosonic Systems, Science 338, 1604 (2012).

[30] X. Chen, Z.-C. Gu, Z.-X. Liu, and X.-G. Wen, Symmetry Protected Topological Orders and the Group Cohomology of Their Symmetry Group, Phys. Rev. B 87, 155114 (2013).

[31] W. J. L. Buyers, R. M. Morra, R. L. Armstrong, M. J. Hogan, P. Gerlach, and K. Hirakawa, Experimental Evidence for the Haldane Gap in a Spin-1 Nearly Isotropic, Antiferromagnetic Chain, Phys. Rev. Lett. 56, 371 (1986).

[32] A. Kitaev, Periodic Table for Topological Insulators and Superconductors, AIP Conf. Proc. 1134, 22 (2009).

[33] A. P. Schnyder, S. Ryu, A. Furusaki, and A. W. W. Ludwig, Classification of Topological Insulators and Superconductors in Three Spatial Dimensions, Phys. Rev. B 78, 195125 (2008).

[34] Z. Bi, A. Rasmussen, K. Slagle, and C. Xu, Classification and Description of Bosonic Symmetry Protected Topological Phases with Semiclassical Nonlinear Sigma Models, Phys. Rev. B 91, 134404 (2015).

[35] L. Fidkowski and A. Kitaev, Effects of Interactions on the Topological Classification of Free Fermion Systems, Phys. Rev. B 81, 134509 (2010).

[36] Z.-C. Gu and M. Levin, Effect of Interactions on TwoDimensional Fermionic Symmetry-Protected Topological Phases with $Z_{2}$ Symmetry, Phys. Rev. B 89, 201113 (2014).

[37] C. Wang, A. C. Potter, and T. Senthil, Classification of Interacting Electronic Topological Insulators in Three Dimensions, Science 343, 629 (2014).

[38] Z.-C. Gu and X.-G. Wen, Symmetry-Protected Topological Orders for Interacting Fermions: Fermionic Topological 
Nonlinear $\sigma$ Models and a Special Group Supercohomology Theory, Phys. Rev. B 90, 115141 (2014).

[39] M. Cheng, Z. Bi, Y.-Z. You, and Z.-C. Gu, Towards a Complete Classification of Symmetry-Protected Phases for Interacting Fermions in Two Dimensions, arXiv: 1501.01313.

[40] P. Bonderson and C. Nayak, Quasi-topological Phases of Matter and Topological Protection, Phys. Rev. B 87, 195451 (2013).

[41] D. A. Huse, R. Nandkishore, V. Oganesyan, A. Pal, and S. L. Sondhi, Localization-Protected Quantum Order, Phys. Rev. B 88, 014206 (2013).

[42] B. Bauer and C. Nayak, Area Laws in a Many-Body Localized State and Its Implications for Topological Order, J. Stat. Mech. (2013) P09005.

[43] Y. Bahri, R. Vosk, E. Altman, and A. Vishwanath, Localization and Topology Protected Quantum Coherence at the Edge of Hot Matter, Nat. Commun. 6, 7341 (2015).

[44] X. Wan, A. M. Turner, A. Vishwanath, and S. Y. Savrasov, Topological Semimetal and Fermi-Arc Surface States in the Electronic Structure of Pyrochlore Iridates, Phys. Rev. B 83, 205101 (2011).

[45] B. Q. Lv, H. M. Weng, B. B. Fu, X. P. Wang, H. Miao, J. Ma, P. Richard, X. C. Huang, L. X. Zhao, G. F. Chen, Z. Fang, X. Dai, T. Qian, and H. Ding, Experimental Discovery of Weyl Semimetal TaAs, Phys. Rev. X 5, 031013 (2015).

[46] L. Lu, Z. Wang, D. Ye, L. Ran, L. Fu, J. D. Joannopoulos, and M. Soljačić, Experimental Observation of Weyl Points, Science 349, 622 (2015).

[47] S.-Y. Xu, I. Belopolski, N. Alidoust, M. Neupane, G. Bian, C. Zhang, R. Sankar, G. Chang, Z. Yuan, C.-C. Lee et al., Discovery of a Weyl Fermion Semimetal and Topological Fermi Arcs, Science 349, 613 (2015).

[48] G. E. Volovik, The Universe in a Helium Droplet (Oxford University Press on Demand, New York, 2003), Vol. 117.

[49] L. Fidkowski, R. M. Lutchyn, C. Nayak, and M. P. A. Fisher, Majorana Zero Modes in One-Dimensional Quantum Wires without Long-Ranged Superconducting Order, Phys. Rev. B 84, 195436 (2011).

[50] J. D. Sau, B. I. Halperin, K. Flensberg, and S. Das Sarma, Number Conserving Theory for Topologically Protected Degeneracy in One-Dimensional Fermions, Phys. Rev. B 84, 144509 (2011).

[51] J. Ruhman, E. Berg, and E. Altman, Topological States in a One-Dimensional Fermi Gas with Attractive Interaction, Phys. Rev. Lett. 114, 100401 (2015).

[52] A. Keselman and E. Berg, Gapless Symmetry-Protected Topological Phase of Fermions in One Dimension, Phys. Rev. B 91, 235309 (2015).

[53] Y. Baum, T. Posske, I. C. Fulga, B. Trauzettel, and A. Stern, Coexisting Edge States and Gapless Bulk in Topological States of Matter, Phys. Rev. Lett. 114, 136801 (2015).

[54] S. Matsuura, P.-Y. Chang, A. P. Schnyder, and S. Ryu, Protected Boundary States in Gapless Topological Phases, New J. Phys. 15, 065001 (2013).

[55] Y. Baum, T. Posske, I. C. Fulga, B. Trauzettel, and A. Stern, Gapless Topological Superconductors: Model Hamiltonian and Realization, Phys. Rev. B 92, 045128 (2015).
[56] D. Pesin and L. Balents, Mott Physics and Band Topology in Materials with Strong Spin-Orbit Interaction, Nat. Phys. 6, 376 (2010).

[57] H.-C. Jiang, Z.-X. Li, A. Seidel, and D.-H. Lee, Symmetry Protected Topological Luttinger Liquids and the Phase Transition between Them, arXiv:1704.02997.

[58] H. Weimer, String Order in Dipole-Blockaded Quantum Liquids, New J. Phys. 16, 093040 (2014).

[59] L. Savary and L. Balents, Quantum Spin Liquids: A Review, Rep. Prog. Phys. 80, 016502 (2017).

[60] S. H. Simon, E. H. Rezayi, N. R. Cooper, and I. Berdnikov, Construction of a Paired Wave Function for Spinless Electrons at Filling Fraction $\nu=2 / 5$, Phys. Rev. B 75, 075317 (2007).

[61] B. I. Halperin, P. A. Lee, and N. Read, Theory of the HalfFilled Landau Level, Phys. Rev. B 47, 7312 (1993).

[62] D. T. Son, Is the Composite Fermion a Dirac Particle?, Phys. Rev. X 5, 031027 (2015).

[63] X. Chen, Y.-M. Lu, and A. Vishwanath, SymmetryProtected Topological Phases from Decorated Domain Walls, Nat. Commun. 5 (2014).

[64] L. Zhang and F. Wang, Unconventional Surface Critical Behavior Induced by a Quantum Phase Transition from the Two-Dimensional Affleck-Kennedy-Lieb-Tasaki Phase to a Néel-Ordered Phase, Phys. Rev. Lett. 118, 087201 (2017).

[65] Y.-M. Lu, Symmetry Protected Gapless $Z_{2}$ Spin Liquids, Phys. Rev. B 93, 165113 (2016).

[66] S. C. Furuya and M. Oshikawa, Symmetry Protection of Critical Phases and a Global Anomaly in $1+1$ Dimensions, Phys. Rev. Lett. 118, 021601 (2017).

[67] J. C. Bridgeman and D. J. Williamson, Anomalies and Entanglement Renormalization, Phys. Rev. B 96, 125104 (2017).

[68] L. Savary, Quantum Loop States in Spin-Orbital Models on the Honeycomb Lattice, arXiv:1511.01505.

[69] B. Yoshida, Topological Phases with Generalized Global Symmetries, Phys. Rev. B 93, 155131 (2016).

[70] F. D. M. Haldane, $O(3)$ Nonlinear $\sigma$ Model and the Topological Distinction between Integer- and HalfInteger-Spin Antiferromagnets in Two Dimensions, Phys. Rev. Lett. 61, 1029 (1988).

[71] T. Kennedy and H. Tasaki, Hidden Symmetry Breaking and the Haldane Phase in $S=1$ Quantum Spin Chains, Commun. Math. Phys. 147, 431 (1992).

[72] Z. Ringel and S. H. Simon, Hidden Order and Flux Attachment in Symmetry-Protected Topological Phases: A Laughlin-like Approach, Phys. Rev. B 91, 195117 (2015).

[73] R. Chatterjee and A. Zamolodchikov, Local Magnetization in Critical Ising Model with Boundary Magnetic Field, Mod. Phys. Lett. A 09, 2227 (1994).

[74] As always, when discussing symmetry breaking, one should specify how the limits $L \rightarrow \infty$ and $h_{B} \rightarrow 0$ (or $h \rightarrow 0$ ) are taken. Strictly speaking, if the boundary field $h_{B}$ decays slower than $1 / \sqrt{L}$, an edge magnetization can occur in the thermodynamic limit due to the fact that a boundary field is a relevant boundary perturbation [73]. This is mainly irrelevant in practice to distinguish gSPT and gTrivial since 
even a field that decays exponentially with $L$ is enough to produce a magnetization in the gSPT case.

[75] S. R. White, Density-Matrix Formulation for Quantum Renormalization Groups, Phys. Rev. Lett. 69, 2863 (1992).

[76] U. Schollwoeck, The Density-Matrix Renormalization Group in the Age of Matrix Product States, Ann. Phys. (Amsterdam) 326, 96 (2011), Special Issue.

[77] J. L. Cardy, Conformal Invariance and Surface Critical Behavior, Nucl. Phys. B240, 514 (1984).

[78] J. L. Cardy, Boundary Conditions, Fusion Rules and the Verlinde Formula, Nucl. Phys. B324, 581 (1989).

[79] J. L. Cardy, Conformal Invariance and Universality in Finite-Size Scaling, J. Phys. A 17, L385 (1984).

[80] L. Tsui, F. Wang, and D.-H. Lee, Topological Versus Landau-like Phase Transitions, arXiv:1511.07460.

[81] L. Tsui, H.-C. Jiang, Y.-M. Lu, and D.-H. Lee, Quantum Phase Transitions Between a Class of Symmetry Protected Topological States, Nucl. Phys. B896, 330 (2015).

[82] L. Tsui, Y.-T. Huang, H.-C. Jiang, and D.-H. Lee, The Phase Transitions Between $Z_{n} \times Z_{n}$ Bosonic Topological Phases in $1+1 d$, and a Constraint on the Central Charge for the Critical Points Between Bosonic Symmetry Protected Topological Phases, Nucl. Phys. B919, 470 (2017).

[83] Y. You and Y.-Z. You, Stripe Melting and a Transition Between Weak and Strong Symmetry Protected Topological Phases, Phys. Rev. B 93, 195141 (2016).

[84] D. S. Fisher, Random Transverse Field Ising Spin Chains, Phys. Rev. Lett. 69, 534 (1992).

[85] D.S. Fisher, Random Antiferromagnetic Quantum Spin Chains, Phys. Rev. B 50, 3799 (1994).

[86] D. Pekker, G. Refael, E. Altman, E. Demler, and V. Oganesyan, Hilbert-Glass Transition: New Universality of Temperature-Tuned Many-Body Dynamical Quantum Criticality, Phys. Rev. X 4, 011052 (2014).

[87] R. Vosk and E. Altman, Dynamical Quantum Phase Transitions in Random Spin Chains, Phys. Rev. Lett. 112, 217204 (2014).

[88] R. Vasseur, A. C. Potter, and S. A. Parameswaran, Quantum Criticality of Hot Random Spin Chains, Phys. Rev. Lett. 114, 217201 (2015).

[89] P. Fendley, Parafermionic Edge Zero Modes in $Z_{n}$-Invariant Spin Chains, J. Stat. Mech. (2012) P11020.

[90] A. S. Jermyn, R. S. K. Mong, J. Alicea, and P. Fendley, Stability of Zero Modes in Parafermion Chains, Phys. Rev. B 90, 165106 (2014).

[91] P. Fendley, Strong Zero Modes and Eigenstate Phase Transitions in the XYZ/Interacting Majorana Chain, J. Phys. A 49, 30LT01 (2016).

[92] ITensor Library (version 2.1.1), http://itensor.org.

[93] D. E. Parker, T. Scaffidi, and R. Vasseur, Symmetry Protected Topological Luttinger Liquids from Decorated Domain Walls (unpublished).

[94] T. Giamarchi, Quantum Physics in One Dimension, International Series of Monographs on Physics (Clarendon Press, Oxford, 2003).

[95] Note that the $U(1)$ symmetry also becomes twisted in the process.

[96] I. Affleck, Edge Magnetic Field in the XXZ Spin- $\frac{1}{2}$ Chain, J. Phys. A 31, 2761 (1998).
[97] J. Ashkin and E. Teller, Statistics of Two-Dimensional Lattices with Four Components, Phys. Rev. 64, 178 (1943).

[98] P. Lecheminant, A. O. Gogolin, and A. A. Nersesyan, Criticality in Self-Dual Sine-Gordon Models, Nucl. Phys. B639, 502 (2002).

[99] H. W. J. Blote and B. Nienhuis, Fully Packed Loop Model on the Honeycomb Lattice, Phys. Rev. Lett. 72, 1372 (1994).

[100] J. Kondev, J. de Gier, and B. Nienhuis, Operator Spectrum and Exact Exponents of the Fully Packed Loop Model, J. Phys. A 29, 6489 (1996).

[101] G. H. Wannier, Antiferromagnetism. The Triangular Ising Net, Phys. Rev. 79, 357 (1950).

[102] M. T. Batchelor, H. W. J. Blöte, B. Nienhuis, and C. M. Yung, Critical Behaviour of the Fully Packed Loop Model on the Square Lattice, J. Phys. A 29, L399 (1996).

[103] D. S. Rokhsar and S. A. Kivelson, Superconductivity and the Quantum Hard-Core Dimer Gas, Phys. Rev. Lett. 61, 2376 (1988).

[104] E. Ardonne, P. Fendley, and E. Fradkin, Topological Order and Conformal Quantum Critical Points, Ann. Phys. (Amsterdam) 310, 493 (2004).

[105] E. Fradkin, D. A. Huse, R. Moessner, V. Oganesyan, and S. L. Sondhi, Bipartite Rokhsar-Kivelson Points and Cantor Deconfinement, Phys. Rev. B 69, 224415 (2004).

[106] Eduardo Fradkin, Field Theories of Condensed Matter Physics (Cambridge University Press, Cambridge, England, 2013).

[107] A. Vishwanath, L. Balents, and T. Senthil, Quantum Criticality and Deconfinement in Phase Transitions Between Valence Bond Solids, Phys. Rev. B 69, 224416 (2004).

[108] B. Hsu and E. Fradkin, Dynamical Stability of the Quantum Lifshitz Theory in $2+1$ Dimensions, Phys. Rev. B 87, 085102 (2013).

[109] E. Fradkin and S. H. Shenker, Phase Diagrams of Lattice Gauge Theories with Higgs Fields, Phys. Rev. D 19, 3682 (1979).

[110] For fully packed loops on the square lattice, one can use a mapping onto a six-vertex model with anisotropy parameter $\Delta=-\frac{1}{2}$, which can be described by a free boson CFT with parameter $g=\frac{1}{3}$. This theory has an additional symmetry $\varphi \rightarrow \varphi+\pi$ due to the bipartite nature of $A$ that corresponds to flipping every other spin on the $A$ lattice.

[111] M. Mulligan, C. Nayak, and S. Kachru, Isotropic to Anisotropic Transition in a Fractional Quantum Hall State, Phys. Rev. B 82, 085102 (2010).

[112] D. Poilblanc, N. Schuch, and I. Affleck, SU(2) Chiral Edge Modes of a Critical Spin Liquid, Phys. Rev. B 93, 174414 (2016).

[113] M. Barkeshli, M. Mulligan, and M. P. A. Fisher, ParticleHole Symmetry and the Composite Fermi Liquid, Phys. Rev. B 92, 165125 (2015).

[114] M. Mulligan, S. Raghu, and M. P. A. Fisher, Emergent Particle-Hole Symmetry in the Half-Filled Landau Level, Phys. Rev. B 94, 075101 (2016).

[115] T. Grover and A. Vishwanath, Quantum Criticality in Topological Insulators and Superconductors: Emergence of Strongly Coupled Majoranas and Supersymmetry, arXiv:1206.1332. 
[116] S. Fubini and C. A. Lutken, Vertex Operators in the Fraction Quantum Hall Effect, Mod. Phys. Lett. A 06, 487 (1991).

[117] G. Moore and N. Read, Nonabelions in the Fractional Quantum Hall Effect, Nucl. Phys. B360, 362 (1991).

[118] B. Blok and X. G. Wen, Many-Body Systems with NonAbelian Statistics, Nucl. Phys. B374, 615 (1992).

[119] C. Nayak, S. H. Simon, A. Stern, M. Freedman, and S. Das Sarma, Non-Abelian Anyons and Topological Quantum Computation, Rev. Mod. Phys. 80, 1083 (2008).

[120] H. Li and F. D. M. Haldane, Entanglement Spectrum as a Generalization of Entanglement Entropy: Identification of Topological Order in Non-Abelian Fractional Quantum Hall Effect States, Phys. Rev. Lett. 101, 010504 (2008).

[121] A. Chandran, M. Hermanns, N. Regnault, and B. A. Bernevig, Bulk-Edge Correspondence in Entanglement Spectra, Phys. Rev. B 84, 205136 (2011).

[122] J. Dubail, N. Read, and E. H. Rezayi, Edge-State Inner Products and Real-Space Entanglement Spectrum of Trial Quantum Hall States, Phys. Rev. B 86, 245310 (2012).

[123] T. Scaffidi and Z. Ringel, Wave Functions of SymmetryProtected Topological Phases from Conformal Field Theories, Phys. Rev. B 93, 115105 (2016).

[124] G. Y. Cho, K. Shiozaki, S. Ryu, and A. W. W. Ludwig, Relationship Between Symmetry Protected Topological Phases and Boundary Conformal Field Theories via the Entanglement Spectrum, J. Phys. A 50, 304002 (2017).

[125] Y.-Z. You, Z. Bi, A. Rasmussen, K. Slagle, and C. Xu, Wave Function and Strange Correlator of Short-Range Entangled States, Phys. Rev. Lett. 112, 247202 (2014).

[126] J. L. Jacobsen, Conformal field theory applied to loop models, in Polygons, Polyominoes and Polycubes (Springer, Netherlands, 2009).

[127] L. Amico, R. Fazio, A. Osterloh, and V. Vedral, Entanglement in Many-Body Systems, Rev. Mod. Phys. 80, 517 (2008).

[128] J. Eisert, M. Cramer, and M. B. Plenio, Colloquium: Area Laws for the Entanglement Entropy, Rev. Mod. Phys. 82, 277 (2010).
[129] E. Fradkin and J.E. Moore, Entanglement Entropy of 2D Conformal Quantum Critical Points: Hearing the Shape of a Quantum Drum, Phys. Rev. Lett. 97, 050404 (2006).

[130] P. Calabrese and J. Cardy, Entanglement Entropy and Quantum Field Theory, J. Stat. Mech. (2004) P06002.

[131] P. Calabrese and J. Cardy, Entanglement Entropy and Conformal Field Theory, J. Phys. A 42, 504005 (2009).

[132] A. B. Zamolodchikov, Irreversibility of the Flux of the Renormalization Group in a $2 D$ Field Theory, JETP Lett. 43, 730 (1986).

[133] B. Ware, J. H. Son, M. Cheng, R. V. Mishmash, J. Alicea, and B. Bauer, Ising Anyons in Frustration-Free MajoranaDimer Models, Phys. Rev. B 94, 115127 (2016).

[134] N. Tarantino and L. Fidkowski, Discrete Spin Structures and Commuting Projector Models for Two-Dimensional Fermionic Symmetry-Protected Topological Phases, Phys. Rev. B 94, 115115 (2016).

[135] M. Hermele, M. P. A. Fisher, and L. Balents, Pyrochlore Photons: The $U(1)$ Spin Liquid in a $S=\frac{1}{2}$ ThreeDimensional Frustrated Magnet, Phys. Rev. B 69 , 064404 (2004).

[136] C. Wang, A. Nahum, and T. Senthil, Topological Paramagnetism in Frustrated Spin-1 Mott Insulators, Phys. Rev. B 91, 195131 (2015).

[137] C. Wang and T. Senthil, Time-Reversal Symmetric U(1) Quantum Spin Liquids, Phys. Rev. X 6, 011034 (2016).

[138] X. G. Wen and A. Zee, Gapless Fermions and Quantum Order, Phys. Rev. B 66, 235110 (2002).

[139] W. Li, S. Yang, M. Cheng, Z.-X. Liu, and H.-H. Tu, Topology and Criticality in the Resonating AffleckKennedy-Lieb-Tasaki Loop Spin Liquid States, Phys. Rev. B 89, 174411 (2014).

[140] J.-M. Stéphan, S. Furukawa, G. Misguich, and V. Pasquier, Shannon and Entanglement Entropies of One- and TwoDimensional Critical Wave Functions, Phys. Rev. B 80, 184421 (2009). 